\title{
Adaptive Backstepping Control for a Class of Uncertain Nonaffine Nonlinear Time-Varying Delay Systems with Unknown Dead-Zone Nonlinearity
}

\author{
Wei-Dong Zhou, Cheng-Yi Liao, and Lan Zheng \\ College of Automation, Harbin Engineering University, Harbin 150001, China \\ Correspondence should be addressed to Cheng-Yi Liao; cugliaocy@163.com
}

Received 19 February 2014; Revised 29 April 2014; Accepted 2 May 2014; Published 21 May 2014

Academic Editor: Gani Stamov

Copyright @ 2014 Wei-Dong Zhou et al. This is an open access article distributed under the Creative Commons Attribution License, which permits unrestricted use, distribution, and reproduction in any medium, provided the original work is properly cited.

An adaptive backstepping controller is constructed for a class of nonaffine nonlinear time-varying delay systems in strict feedback form with unknown dead zone and unknown control directions. To simplify controller design, nonaffine system is first transformed into an affine system by using mean value theorem and the unknown nonsymmetric dead-zone nonlinearity is treated as a combination of a linear term and a bounded disturbance-like term. Owing to the universal approximation property, fuzzy logic systems (FLSs) are employed to approximate the uncertain nonlinear part in controller design process. By introducing Nussbaumtype function, the a priori knowledge of the control gains signs is not required. By constructing appropriate Lyapunov-Krasovskii functionals, the effect of time-varying delay is compensated. Theoretically, it is proved that this scheme can guarantee that all signals in closed-loop system are semiglobally uniformly ultimately bounded (SUUB) and the tracking error converges to a small neighbourhood of the origin. Finally, the simulation results validate the effectiveness of the proposed scheme.

\section{Introduction}

In the past decade, adaptive backstepping design technique has received a great deal of attention since it was pioneered by Kanellakopoulos et al. in 1991 [1]. In [2-4], adaptive backstepping is utilized to construct robust adaptive backstepping controller. The main feature of this approach is that it can handle nonlinear systems without satisfying the matching conditions, but the backstepping design procedure has a shortcoming named explosion of complexity because of the repeated differentiations of virtual controllers. By using dynamic surface control technique, the explosion of complexity shortcoming is overcome [5]. References $[6,7]$ develop a command filtered backstepping approach which is feasible even when the number of iterations of the backstepping method is large. However, it should be noted that the nonlinear functions are all assumed to be known in the abovementioned methods. Recently, many adaptive backstepping controllers with FLSs or neural networks (NNs) have been developed for nonlinear systems in strict feedback form [8-27]. Owing to the universal approximation property of FLSs or NNs, these control approaches do not require the precise knowledge of system nonlinearities. Nevertheless, the introduced FLSs or NNs may lead to a burdensome computation when the number of the parameters which need to be tuned by online learning laws increases significantly. To handle the inevitable weakness meeting when increasing the number of fuzzy rules or neural network nodes, the optimal weighting vector in FLSs is used as the estimation parameter [8,9]. In $[10,14,19,21,25,27]$, FLSs are utilized to directly approximate the desired control signals instead of the unknown nonlinearities in each backstepping design step. Consequently, the number of parameters needed to be adapted is significantly reduced for only one parameter needed to be estimated online no matter how many fuzzy rules are selected. On the basis of the work in [10], a novel adaptive fuzzy backstepping controller construct method without requirement of the fuzzy basis functions is exploited $[22,23]$.

Dead-zone characteristic is one of the most common actuator nonsmooth nonlinearities encountered in many industrial processes, which can seriously affect the system 
performance and indeed make the system unstable. Many controller design schemes are developed for systems with unknown dead zone [2, 3, 15-17, 28-36]. Generally, the dead zone is first treated as a combination of a linear and a bounded disturbance-like term, and then the controller that can achieve a good control performance is designed by adopting robust control technique [16, 17, 28-31]. In [32], a novel two-layered fuzzy logic controller which consists of a fuzzy logic-based precompensator and a usual fuzzy PD controller are developed for controlling systems with dead zone. In $[33,34]$, by introducing a fuzzy logic deadzone compensator two fuzzy controllers are constructed for motion control system and a DC motor system, respectively. Nevertheless, when there are no suitable rules for the deadzone nonlinearity, this method may be unfeasible for it depends much on operators or experts experience. In $[2,3$, $15,35,36]$, the inverse function of dead zone is utilized to compensate the effect of the dead zone. Using this method, an effective control has been achieved, but the shortcoming that the dead-zone parameters are required to be constants is inevitable. Regrettably, although much progress has been made in the fields of controller design for nonlinear systems with unknown dead zone, nonaffine nonlinear systems with unknown dead zone are seldomly investigated.

Time delays frequently occur in practical control systems, such as electrical networks and hydraulic systems. Considering that the existing time delays often cause system instability and performance deterioration, to handle the control problem for systems with time delays is an unavoidable issue. Two main tools Lyapunov-Krasovskii functionals and Lyapunov-Razumikhin functions are usually applied to nonlinear time-delay systems $[4,17-25,37-39]$. In [17-19, 22-24], Lyapunov-Krasovskii functionals are constructed to compensate the unknown time delays. Within these schemes, the condition that the unknown time delays are assumed to be unknown constants is too strict. To solve time-varying delays problem, a novel Lyapunov-Krasovskii functionals are designed on condition that the derivative of time delay functions is less than one $[20,25,30,37]$. In $[4,21,38]$, Lyapunov-Razumikhin lemma-based adaptive backstepping control approaches are proposed for nonlinear systems in which the limitation condition on the derivative of time delay is cancelled. In $[17,24,25]$, adaptive fuzzy or neural backstepping controllers are designed for a class of nonlinear time-delay systems with unknown control directions. As control direction, that is, the sign of control gain, decide the direction along which the controller parameters are updated, designing adaptive controllers for these unknown systems with the control direction becoming much more difficult. Nussbaum-type function is utilized to deal with the unknown control direction $[17,24,25]$. A robust adaptive NNs controller is first proposed for a class of nonlinear timedelay systems with unknown dead-zone nonlinearity and unknown control direction [17]. However, in this method, the time delay is supposed to be unknown constants and the NNs introduced to approximate the uncertain nonlinear term may result in complexity computation when the dimension of system increases.
Inspired by the preceding discussion, in this paper, a class of nonaffine nonlinear time-varying delay systems with both unknown dead-zone input and completely unknown control direction is investigated and an adaptive fuzzy backstepping control scheme is exploited. The main contributions of this paper can be summarized as follows. (1) Few papers consider nonaffine systems with unknown dead-zone nonlinearity. The difficulty of design controller for nonaffine systems is that the control input appears nonlinear in unknown nonlinear systems. Mean value theorem is used to transform the nonaffine form into an affine form, and then the existing approaches for affine systems can be directly applied [40]. (2) Similar to [10], FLSs are directly employed to approximate the unknown nonlinearities. Considering the norm of the ideal weighting vector in FLSs as the estimation parameter instead of the elements of weighting vector, there is only one parameter that needs to be estimated online in each step. Meanwhile, it should be noted that in this control approach, the basic functions of FLSs do not occur in the control laws and adaptive laws. This improvement can overcome the explosion of complexity caused by repeated differentiations of virtual controllers and the increase of system dimension. (3) The other encountered trouble is how to cope with the unknown time delay terms in system. Compared with [17], the time delay term considered in this paper is time varying and the novel Lyapunov-Krasovskii functionals are employed to stability analysis and synthesis. In particular, here, the reason we use Lyapunov-Krasovskii functionals to construct controller is that this method can provide less conservative and delay-independent results. Using the Lyapunov stability theorem, it is proved that the proposed control schemes can guarantee that all the signals in closedloop system are bounded and the tracking error is asymptotic convergence. Finally, effectiveness of the developed scheme is demonstrated by the simulation examples.

\section{Problem Formulation and Preliminaries}

Consider the following SISO nonaffine nonlinear timevarying delay system:

$$
\begin{aligned}
\dot{x}_{i}(t)= & f_{i}\left(\overline{\mathbf{x}}_{i}(t)\right)+g_{i}\left(\overline{\mathbf{x}}_{i}(t)\right) x_{i+1}(t)+d_{i}(t) \\
& +h_{i}\left(x_{1}\left(t-\tau_{1}(t)\right), \ldots, x_{i}\left(t-\tau_{i}(t)\right)\right), \\
\dot{x}_{n}(t)= & f(\mathbf{x}(t), D(u(t)))+d_{n}(t) \\
& +h_{n}\left(x_{1}\left(t-\tau_{1}(t)\right), \ldots, x_{n}\left(t-\tau_{n}(t)\right)\right), \\
& y(t)=x_{1}(t), \quad i=1, \ldots, n-1,
\end{aligned}
$$

where $u \in R$ and $y \in R$ are the system control input and output, respectively, $\overline{\mathbf{x}}_{i}(t)=\left[x_{1}(t), \ldots, x_{i}(t)\right]^{T}$ and $\mathbf{x}(t)=$ $\left[x_{1}(t), \ldots, x_{n}(t)\right]^{T}$ are system states, $f_{i}(\cdot), g_{i}(\cdot)$, and $f(\cdot)$ are unknown smooth functions, and $i=1, \ldots, n-1 . h_{i}(\cdot)$ is an unknown smooth function with the unknown time-varying delay terms $\tau_{i}(t)$. There exists a positive constant $\tau_{0}$ satisfying $0 \leq \tau_{i}(t) \leq \tau_{0}<+\infty$, and $\varphi_{i}(s)=x_{i}(s), \forall s \in\left[-\tau_{0}, 0\right]$ is an unknown continuous bounded initial function. $d_{i}(t)$ denotes 
the unknown external disturbance, $i=1, \ldots, n . D(u(t))$ is the unknown dead-zone input.

The control objective is to design an adaptive backstepping controller for system (1) such that the system output $y(t)$ tracks the desired trajectory $y_{d}(t)$ and all signals in closedloop system are bounded.

Utilizing the mean value theorem [40], function $f(\cdot)$ in (1) can be rewritten as

$$
\begin{aligned}
f & (\mathbf{x}(t), D(u(t))) \\
& =f(\mathbf{x}(t), 0)+\left.\frac{\partial f(\mathbf{x}(t), D(u(t)))}{\partial D(u(t))}\right|_{D(u)=\lambda} D(u(t)) \\
& =f_{n}(\mathbf{x}(t))+g_{n}(\mathbf{x}(t)) D(u(t)),
\end{aligned}
$$

with $f_{n}(\mathbf{x}(t))=f(\mathbf{x}(t), 0)$ and $g_{n}(\mathbf{x}(t))=$ $\partial f(\mathbf{x}(t), D(u(t))) /\left.\partial D(u(t))\right|_{D(u(t))=\lambda} ; \lambda \in(0, D(u))$.

Assumption 1. There exist constants $g$ and $\bar{g}$ satisfying $0<$ $\underline{g} \leq\left|g_{i}\left(\overline{\mathbf{x}}_{i}(t)\right)\right| \leq \bar{g}$.

The nonsymmetric dead-zone input is defined as [17]

$$
D(u(t))= \begin{cases}\varphi_{r}(t)\left(u(t)-m_{r}\right), & u(t)>m_{r}, \\ 0, & -m_{l} \leq u(t) \leq m_{r}, \\ \varphi_{l}(t)\left(u(t)+m_{l}\right), & u(t)<-m_{l},\end{cases}
$$

where $\varphi_{r}(t)$ and $\varphi_{l}(t)$ are unknown right and left slopes of the dead zone and $m_{r}$ and $m_{l}$ are breakpoints of the dead zone. To deal with dead-zone nonlinearity, the following assumptions are put forward.

Assumption 2. $m_{r}$ and $m_{l}$ are unknown bounded constants. $\varphi_{r}(t)$ and $\varphi_{l}(t)$ are unknown functions and there are unknown positive constants $\varphi_{r 0}, \varphi_{r 1}, \varphi_{l 0}$, and $\varphi_{l 1}$ satisfying

$$
\begin{aligned}
& 0<\varphi_{r 0} \leq \varphi_{r}(t) \leq \varphi_{r 1}, \\
& 0<\varphi_{l 0} \leq \varphi_{l}(t) \leq \varphi_{l 1} .
\end{aligned}
$$

Define vectors $\boldsymbol{\eta}(t)$ and $\boldsymbol{\kappa}(t)$ as

$$
\begin{aligned}
\boldsymbol{\eta}(t) & =\left[\eta_{r}(t), \eta_{l}(t)\right]^{T}, \\
\boldsymbol{\kappa}(t) & =\left[\varphi_{r}(t), \varphi_{l}(t)\right]^{T},
\end{aligned}
$$

with $\eta_{r}(t)=\left\{\begin{array}{l}1, u(t) \geq-m_{l} \\ 0, u(t)<-m_{l}\end{array}\right.$ and $\eta_{l}(t)=\left\{\begin{array}{l}1, u(t) \leq m_{r} \\ 0, u(t)>m_{r}\end{array}\right.$.

Based on the above analysis, the dead zone can be expressed as

$$
D(u)=\boldsymbol{\eta}^{T}(t) \boldsymbol{\kappa}(t) u(t)+d(u(t))
$$

where

$$
\begin{aligned}
& d(u(t)) \\
& \quad= \begin{cases}-\varphi_{r}(t) m_{r}, & u(t) \geq m_{r}, \\
-\left(\varphi_{r}(t)+\varphi_{l}(t)\right) u(t), & -m_{l}<u(t)<m_{r}, \\
\varphi_{l}(t) m_{l}, & u(t) \leq-m_{l} .\end{cases}
\end{aligned}
$$

According to Assumption 2, it can be concluded that $|d(u(t))| \leq d_{0}$ and $d_{0}$ is an unknown positive constant meeting $d_{0}=\left(\varphi_{r 1}+\varphi_{l 1}\right) \max \left\{m_{r}, m_{l}\right\}$. Considering the definition of $\boldsymbol{\eta}(t)$ and $\boldsymbol{\kappa}(t)$, we have

$$
\boldsymbol{\eta}^{T}(t) \boldsymbol{\kappa}(t)= \begin{cases}\varphi_{r}(t), & u(t) \geq m_{r}, \\ \varphi_{r}(t)+\varphi_{l}(t), & -m_{l}<u(t)<m_{r}, \\ \varphi_{l}(t), & u(t) \leq-m_{l} .\end{cases}
$$

It is easy to obtain that $\boldsymbol{\eta}^{T}(t) \boldsymbol{\kappa}(t) \in\left[\min \left(\varphi_{r 0}, \varphi_{l 0}\right), \varphi_{r 1}+\right.$ $\left.\varphi_{l 1}\right]$; that is, $\boldsymbol{\eta}^{T}(t) \boldsymbol{\kappa}(t)$ is a positive discontinuous bounded function.

Using (2) and (6), system (1) can be transformed into the following form:

$$
\begin{gathered}
\dot{x}_{i}(t)=f_{i}\left(\overline{\mathbf{x}}_{i}(t)\right)+g_{i}\left(\overline{\mathbf{x}}_{i}(t)\right) x_{i+1}(t)+d_{i}(t) \\
+h_{i}\left(x_{1}\left(t-\tau_{1}(t)\right), \ldots, x_{i}\left(t-\tau_{i}(t)\right)\right), \\
\dot{x}_{n}(t)=f_{n}(\mathbf{x})+g_{n}(\mathbf{x}) \boldsymbol{\eta}^{T}(t) \boldsymbol{\kappa}(t) u(t)+g_{n}(\mathbf{x}) d(u(t)) \\
+h_{n}\left(x_{1}\left(t-\tau_{1}(t)\right), \ldots, x_{n}\left(t-\tau_{n}(t)\right)\right)+d_{n}(t), \\
y(t)=x_{1}(t) .
\end{gathered}
$$

Notation. $x_{i}, x_{i}\left(\tau_{i}\right), y, u, d_{i}, f_{i}$, and $g_{i}$ denote $x_{i}(t), x_{i}\left(t-\tau_{i}(t)\right)$, $y(t), u(t), d_{i}(t), f_{i}\left(\overline{\mathbf{x}}_{i}\right)$, and $g_{i}\left(\overline{\mathbf{x}}_{i}\right)$, respectively.

Assumption 3. The unknown smooth functions $h_{i}\left(x_{1}\left(\tau_{1}\right), \ldots\right.$, $\left.x_{i}\left(\tau_{i}\right)\right)$ satisfy the inequality

$$
\left|h_{i}\left(x_{1}\left(\tau_{1}\right), \ldots, x_{i}\left(\tau_{i}\right)\right)\right| \leq \sum_{j=1}^{i} H_{i, j}\left(x_{j}\left(\tau_{j}\right)\right)
$$

where $H_{i, j}(\cdot)$ are unknown positive smooth functions.

Remark 4. Compared with [17] in which the bounding functions are required to be known, it should be emphasized that the nonlinear functions $H_{i, j}(\cdot)$ are unknown in this paper.

Assumption 5. The time derivatives of the time-varying delay terms $\tau_{i}$ are $\dot{\tau}_{i}$ and satisfy $\dot{\tau}_{i} \leq \tau^{*}<1$ where $\tau^{*}$ is an unknown positive constant.

Assumption 6. The external disturbances $d_{i}$ satisfy $\left|d_{i}\right| \leq \bar{d}_{i}$, where $\bar{d}_{i}$ is defined as an unknown positive constant.

Remark 7. The constants $g_{i 0}, g_{i 1}, \tau_{0}, \tau^{*}$, and $\bar{d}_{i}$ are only required for analytical purposes and their values are not necessarily known in control laws and adaptive laws.

Before we derive our results, the FLSs and Nussbaumtype function should be introduced.

The FLS has a basic configuration which contains fuzzifier, fuzzy rule base, fuzzy reference engine, and defuzzifier, 
such four components. The fuzzy rule base is composed of a series If-Then inference rules in the following form [41]:

$$
\begin{array}{r}
R^{(l)}: \text { If } x_{1} \text { is } F_{1}^{l} \text { and } \cdots \text { and } x_{n} \text { is } F_{n}^{l}, \\
\text { Then } y \text { is } G, \quad l=1, \ldots, M,
\end{array}
$$

where $\mathbf{x}=\left[x_{1}, x_{2}, \ldots, x_{n}\right]^{T}$ and $y$ are the FLS inputs and output, respectively. $F_{i}^{l}, i=1, \ldots, n$, and $G^{l}$ are fuzzy sets characterized by fuzzy membership functions $\mu_{F_{i}^{l}}\left(x_{i}\right)$ and $\mu_{G^{l}}(y)$, respectively, and $M$ is the number of fuzzy rule. The final output of the fuzzy system can be expressed by using the singleton fuzzifier, product inference engine, and centeraverage defuzzifier as follows [41]:

$$
y(\mathbf{x})=\frac{\sum_{l=1}^{M} \bar{y}^{l}\left(\prod_{i=1}^{n} \mu_{F_{i}^{l}}\left(x_{i}\right)\right)}{\sum_{l=1}^{M}\left(\prod_{i=1}^{n} \mu_{F_{i}^{l}}\left(x_{i}\right)\right)},
$$

where $\bar{y}^{l}$ is the point at which the membership function $\mu_{G^{l}}(y)$ achieves its maximum value and we assume that $\mu_{G^{l}}\left(\bar{y}^{l}\right)=1$. Let $\boldsymbol{\theta}=\left[\bar{y}^{1}, \ldots, \bar{y}^{M}\right]^{T}$ be a vector grouping all consequent parameters and $\xi(\mathbf{x})=\left[\xi_{1}(\mathbf{x}), \ldots, \xi_{M}(\mathbf{x})\right]^{T}$, where $\xi_{j}(\mathbf{x}) \triangleq \prod_{i=1}^{n} \mu_{F_{i}^{j}}\left(x_{i}\right) / \sum_{l=1}^{M}\left(\prod_{i=1}^{n} \mu_{F_{i}^{l}}\left(x_{i}\right)\right), j=1,2, \ldots, M$ is the vector of fuzzy basis function. Then, using the conception of fuzzy basis functions [41], the output of the fuzzy logic system can be formulated as $y(\mathbf{x})=\boldsymbol{\theta}^{T} \xi(\mathbf{x})$. Then according to the universal approximation theorem, any continuous nonlinear function $f(\mathbf{x})$ can be approximated by the FLS as

$$
f(\mathbf{x})=\boldsymbol{\theta}_{f}^{* T} \xi(\mathbf{x})+\varepsilon(\mathbf{x}),
$$

where $\boldsymbol{\theta}_{f}^{*}$ is an optimal parameter satisfying $\boldsymbol{\theta}_{f}^{*}=$ $\arg \min _{\boldsymbol{\theta}_{f}}\left(\sup _{x \in \mathbf{x}}|\widehat{f}(\mathbf{x})-f(\mathbf{x})|\right)$ and $\varepsilon(\mathbf{x})$ is the minimum approximation error satisfying $|\varepsilon(\mathbf{x})| \leq b_{0}$ ( $b_{0}$ is a positive constant).

Nussbaum-type function is successfully applied to cope with the problem caused by unknown control direction [17, $24,25,27]$. A function which has the following properties is called Nussbaum function [42]:

$$
\begin{aligned}
& \lim _{k \rightarrow \infty} \sup \frac{1}{k} \int_{0}^{k} N(\varsigma) d \varsigma=+\infty, \\
& \lim _{k \rightarrow \infty} \inf \frac{1}{k} \int_{0}^{k} N(\varsigma) d \varsigma=-\infty .
\end{aligned}
$$

Functions, such as $\varsigma^{2} \cos (\varsigma), \varsigma^{2} \sin (\varsigma)$ and $\exp \left(\varsigma^{2}\right)$ $\cos ((\pi / 2) \varsigma)$, are commonly used as Nussbaum functions for nonlinear systems with unknown control direction. In this paper, the Nussbaum function $N(\varsigma)=\varsigma^{2} \cos (\varsigma)$ is employed.

Lemma 8 (see [17]). $V(t)$ and $\varsigma(t)$ are smooth functions with $V(t) \geq 0$. If the inequality $V(t) \leq c_{0}+e^{-c_{1} t} \int_{0}^{t}[g(x(\tau)) N(\varsigma)+$ $1] \dot{\zeta} e^{c_{1} \tau} d \tau$ holds with $t \in\left[0, t_{f}\right), c_{0}$ is a suitable constant, $N(\varsigma)=$ $\varsigma^{2} \cos (\varsigma), c_{1}$ is a positive constant, and $g(x)$ is a time-varying parameter which takes values in unknown closed intervals $I=$ $[\underline{g}, \bar{g}]$ and $0 \notin I$, then $V(t), \varsigma(t)$, and $\int_{0}^{t} g(x(\tau)) N(\varsigma) \dot{\zeta} d \tau$ must be bounded on $\left[0, t_{f}\right)$.

\section{Controller Design and Stability Analysis}

In this section, an adaptive fuzzy control scheme is presented by using backstepping technique combined with LyapunovKrasovskii functionals and Nussbaum type functions. The backstepping design is based on the following change of coordinates:

$$
\begin{gathered}
\chi_{1}=x_{1}-y_{d} \\
\chi_{i}=x_{i}-\alpha_{i-1}, \quad i=2, \ldots, n,
\end{gathered}
$$

where $\alpha_{i-1}$ is a virtual control which should be designed for the corresponding $(i-1)$ th subsystem. In general, the design procedure contains $n$ steps. FLSs are employed to approximate the unknown nonlinear term. Then, let us define unknown constants satisfying

$$
\vartheta_{i}=\left\|\boldsymbol{\theta}_{i}\right\|^{2}, \quad i=1, \ldots, n \text {. }
$$

For $\vartheta_{i}$ are unknown and $\widehat{\vartheta}_{i}$ are used to estimate $\vartheta_{i}$ with estimation errors $\widetilde{\vartheta}_{i}$ defined as $\widetilde{\vartheta}_{i}=\vartheta_{i}-\widehat{\vartheta}_{i}$.

The detailed design procedure is described in the following steps.

Step 1. Consider the Lyapunov-Krasovskii function as

$$
V_{1}=\frac{1}{2} \chi_{1}^{2}+\frac{1}{2 \gamma_{1}} \widetilde{\vartheta}_{1}^{2}+V_{1,0},
$$

where $V_{1,0}=\left(e^{-r\left(t-\tau_{0}\right)} / 1-\tau^{*}\right) \int_{t-\tau_{1}(t)}^{t} e^{r s} H_{1,1}^{2}\left(x_{1}(s)\right) d s, \gamma_{1}$ and $r$ are design positive parameters.

Giving a compact set $\Omega_{\kappa_{1}}$ as $\Omega_{\kappa_{1}}=\left\{\chi_{1}|| \chi_{1} \mid<0.2554 \kappa_{1}\right\}$ with $\kappa_{1}$, a positive design parameter, then a function defined as follows will be employed to design controller

$$
q_{1}\left(\chi_{1}\right)= \begin{cases}1, & \chi_{1} \notin \Omega_{\kappa_{1}} \\ 0, & \chi_{1} \in \Omega_{\kappa_{1}} .\end{cases}
$$

We choose the virtual control law $\alpha_{1}$ and adaptive laws as

$$
\begin{gathered}
\alpha_{1}=\frac{q_{1}\left(\chi_{1}\right)}{2 a_{1}^{2}} N\left(\varsigma_{1}\right) \chi_{1} \widehat{\vartheta}_{1}, \\
\dot{\varsigma}_{1}=\frac{q_{1}\left(\chi_{1}\right)}{2 a_{1}^{2}} \chi_{1}^{2} \widehat{\vartheta}_{1}, \\
\dot{\widehat{\vartheta}}_{1}=\frac{\gamma_{1}}{2 a_{1}^{2}} \chi_{1}^{2}-\eta_{1} \widehat{\vartheta}_{1},
\end{gathered}
$$

where $a_{1}$ and $\eta_{1}$ are design positive parameters.

Case 1. In this case, we have $\left|\chi_{1}\right|<0.2554 \kappa_{1}$. Apparently, the tracking error $\chi_{1}$ is bounded. According to (18)-(20), we can conclude that when we select bounded initial values, $\alpha_{1}$ is bounded and $\dot{\zeta}_{1}=0$; that is, $N\left(\varsigma_{1}\right)$ is bounded. Integrating (21) over $[0, t]$ we get that signal $\widehat{\vartheta}_{1}$ is bounded.

Case 2. When $\left|\chi_{1}\right| \geq 0.2554 \kappa_{1}$, the following process is needed. 
The time derivative of $V_{1}$ is

$$
\begin{aligned}
\dot{V}_{1} \leq & \chi_{1}\left(f_{1}+g_{1} x_{2}+h_{1}\left(x_{1}\left(\tau_{1}\right)\right)+d_{1}-\dot{y}_{d}\right) \\
& -\frac{1}{\gamma_{1}} \widetilde{\vartheta}_{1} \dot{\hat{\vartheta}}_{1}-r V_{1,0} \\
& +\frac{e^{-r\left(t-\tau_{0}\right)}}{1-\tau^{*}}\left[e^{r t} H_{1,1}^{2}\left(x_{1}\right)\right. \\
& \left.\quad-\left(1-\dot{\tau}_{1}(t)\right) e^{r\left(t-\tau_{1}(t)\right)} H_{1,1}^{2}\left(x_{1}\left(\tau_{1}\right)\right)\right] \\
\leq & \chi_{1}\left(f_{1}+g_{1} x_{2}+h_{1}\left(x_{1}\left(\tau_{1}\right)\right)+d_{1}-\dot{y}_{d}\right) \\
& -\frac{1}{\gamma_{1}} \widetilde{\vartheta}_{1} \dot{\hat{\vartheta}}_{1}-r V_{1,0}+H_{1}\left(x_{1}\right)-H_{1,1}^{2}\left(x_{1}\left(\tau_{1}\right)\right),
\end{aligned}
$$

with $H_{1}\left(x_{1}\right)=e^{r \tau_{0}} H_{1,1}^{2}\left(x_{1}\right) /\left(1-\tau^{*}\right)$.

When Assumption 3 holds, we get

$$
\left|h_{1}\left(x_{1}\left(\tau_{1}\right)\right)\right|^{2} \leq H_{1,1}^{2}\left(x_{1}\left(\tau_{1}\right)\right) \text {. }
$$
yields

By using Young's inequality and combining with (23), (22)

$$
\begin{aligned}
\dot{V}_{1}= & \chi_{1} g_{1}\left(\chi_{2}+\alpha_{1}\right)+\chi_{1}\left(f_{1}+d_{1}-\dot{y}_{d}\right) \\
& +\frac{1}{4} \chi_{1}^{2}+h_{1}^{2}\left(x_{1}\left(\tau_{1}\right)\right)-\frac{1}{\gamma_{1}} \widetilde{\vartheta}_{1} \dot{\hat{\vartheta}}_{1} \\
& -r V_{1,0}+H_{1}\left(x_{1}\right)-H_{1,1}^{2}\left(x_{1}\left(\tau_{1}\right)\right) \\
\leq & -c_{1} \chi_{1}^{2}+\chi_{2}^{2}+\chi_{1} g_{1} \alpha_{1} \\
& +\chi_{1}\left(f_{1}-\dot{y}_{d}+c_{1} \chi_{1}+\frac{1}{2 \rho^{2}} \chi_{1}+\frac{1}{4} g_{1}^{2} \chi_{1}+\frac{3}{4} \chi_{1}\right) \\
& -\frac{1}{2} \chi_{1}^{2}+\frac{1}{2} \rho^{2} \bar{d}_{1}^{2}-\frac{1}{\gamma_{1}} \widetilde{\vartheta}_{1} \dot{\hat{\vartheta}}_{1}+H_{1,1}^{2}\left(x_{1}\left(\tau_{1}\right)\right) \\
& -r V_{1,0}+H_{1}\left(x_{1}\right)-H_{1,1}^{2}\left(x_{1}\left(\tau_{1}\right)\right) \\
= & -c_{1} \chi_{1}^{2}-r V_{1,0}+\chi_{2}^{2}+\chi_{1} g_{1} \alpha_{1}+\chi_{1} \overline{f_{1}} \\
& -\frac{1}{\gamma_{1}} \widetilde{\vartheta}_{1} \dot{\hat{\vartheta}} 1+\left(1-16 \tanh _{1}^{2}\left(\frac{\chi_{1}}{\kappa_{1}}\right)\right) H_{1}\left(x_{1}\right) \\
& -\frac{1}{2} \chi_{1}^{2}+\frac{1}{2} \rho^{2} \bar{d}_{1}^{2},
\end{aligned}
$$

where $c_{1}$ and $\rho$ are positive constants

$$
\begin{aligned}
\overline{f_{1}}= & f_{1}-\dot{y}_{d}+c_{1} \chi_{1}+\frac{1}{2 \rho^{2}} \chi_{1}+\frac{1}{4} g_{1}^{2} \chi_{1} \\
& +\frac{3}{4} \chi_{1}+\frac{16}{\chi_{1}} \tanh ^{2}\left(\frac{\chi_{1}}{\kappa_{1}}\right) H_{1}\left(x_{1}\right) .
\end{aligned}
$$

Remark 9. Note that the function $H_{i}\left(x_{i}\right) / \chi_{i}(i=1, \ldots, n)$ is not defined at $\chi_{i}=0$ which leads to the fact that it cannot be approximated by FLSs. To cope with this difficulty, we introduce function $\left(16 / \chi_{i}\right) \tanh ^{2}\left(\chi_{i} / \kappa_{i}\right) H_{i}\left(x_{i}\right)$ instead of $H_{i}\left(x_{i}\right) / \chi_{i}$ according to the effective approach in
[19]. The design parameter $\kappa_{i}$ can be adjusted to achieve better performance.

As $\overline{f_{1}}$ consists of unknown nonlinear functions $f_{1}, g_{1}$, and $H_{1}\left(x_{1}\right), \overline{f_{1}}$ cannot be directly used to construct controller. According to the universal approximation property of FLSs, $\overline{f_{1}}$ can be rewritten as

$$
\overline{f_{1}}=\boldsymbol{\theta}_{1}^{* T} \boldsymbol{\xi}_{1}\left(\mathbf{Z}_{1}\right)+\delta_{1}\left(\mathbf{Z}_{1}\right), \quad\left|\delta_{1}\left(\mathbf{Z}_{1}\right)\right| \leq \varepsilon_{1},
$$

where $\delta_{1}\left(\mathbf{Z}_{1}\right)$ stands for approximation error and $\varepsilon_{1}$ is an unknown constant, $\mathbf{Z}_{1}=\left[x_{1}, y_{d}, \dot{y}_{d}\right]^{T}$.

Considering the inequality $0<\xi_{1}^{T}\left(\mathbf{Z}_{1}\right) \xi_{1}\left(\mathbf{Z}_{1}\right) \leq 1$ we obtain

$$
\begin{aligned}
\chi_{1} \overline{f_{1}} & =\chi_{1} \boldsymbol{\theta}_{1}^{* T} \boldsymbol{\xi}_{1}\left(\mathbf{Z}_{1}\right)+\chi_{1} \delta_{1}\left(\mathbf{Z}_{1}\right) \\
& \leq \frac{1}{2 a_{1}^{2}} \chi_{1}^{2} \vartheta_{1}+\frac{1}{2} a_{1}^{2}+\frac{1}{2} \chi_{1}^{2}+\frac{1}{2} \varepsilon_{1}^{2} .
\end{aligned}
$$

As $\left|\chi_{1}\right| \geq 0.2554 \kappa_{1}$, we have

$$
\left(1-16 \tanh ^{2}\left(\frac{\chi_{1}}{\kappa_{1}}\right)\right) H_{1}\left(x_{1}\right) \leq 0 .
$$

Substituting (27) and (28) into (24) results in

$$
\begin{gathered}
\dot{V}_{1} \leq-c_{1} \chi_{1}^{2}-r V_{1,0}+\chi_{2}^{2}+\chi_{1} g_{1} \alpha_{1}+\frac{1}{2 a_{1}^{2}} \chi_{1}^{2} \vartheta_{1} \\
+\frac{1}{2} a_{1}^{2}+\frac{1}{2} \varepsilon_{1}^{2}+\frac{1}{2} \rho^{2} \bar{d}_{1}^{2}-\frac{1}{\gamma_{1}} \widetilde{\vartheta}_{1} \dot{\widehat{\vartheta}}_{1} .
\end{gathered}
$$

Considering $q\left(\chi_{1}\right)=1$ and using (19)-(21), (29) yields

$$
\begin{aligned}
\dot{V}_{1} \leq & -c_{1} \chi_{1}^{2}-r V_{1,0}+\chi_{2}^{2}+\left[g_{1} N\left(\varsigma_{1}\right)+1\right] \dot{\varsigma}_{1} \\
& +\frac{1}{2} a_{1}^{2}+\frac{1}{2} \varepsilon_{1}^{2}+\frac{1}{2} \rho^{2} \bar{d}_{1}^{2}+\frac{\eta_{1}}{\gamma_{1}} \widetilde{\vartheta}_{1} \widehat{\vartheta}_{1} \\
\leq & -\mu_{1} V_{1}+\chi_{2}^{2}+\left[g_{1} N\left(\varsigma_{1}\right)+1\right] \dot{\varsigma}_{1}+C_{1},
\end{aligned}
$$

with $\mu_{1}=\min \left(2 c_{1}, \eta_{1}, r\right)$ and $C_{1}=(1 / 2) a_{1}^{2}+(1 / 2) \varepsilon_{1}^{2}+$ $(1 / 2) \rho^{2} \bar{d}_{1}^{2}+\left(\eta_{1} / 2 \gamma_{1}\right) \vartheta_{1}^{2}$.

Multiplying (30) by $e^{\mu_{1} t}$ and then integrating over $[0, t]$, we get

$$
\begin{aligned}
V_{1} \leq & \beta_{1}+e^{-\mu_{1} t} \int_{0}^{t}\left[g_{1} N\left(\varsigma_{1}\right)+1\right] \dot{\varsigma}_{1} e^{\mu_{1} \tau} d \tau \\
& +e^{-\mu_{1} t} \int_{0}^{t} \chi_{2}^{2} e^{\mu_{1} \tau} d \tau
\end{aligned}
$$

where $\beta_{1}=\left(C_{1} / \mu_{1}\right)+\left(V_{1}(0)-\left(C_{1} / \mu_{1}\right)\right) e^{-\mu_{1} t}$.

Noting that there is an extra term $e^{-\mu_{1} t} \int_{0}^{t} \chi_{2}^{2} e^{\mu_{1} \tau} d \tau$ within (31), we suppose that $\chi_{2}$ can be regulated as bounded; then we have

$$
\begin{aligned}
e^{-\mu_{1} t} \int_{0}^{t} \chi_{2}^{2} e^{\mu_{1} s} d s & \leq e^{-\mu_{1} t} \sup _{s \in[0, t]}\left(\chi_{2}^{2}(s)\right) \int_{0}^{t} e^{\mu_{1} s} d s \\
& \leq \frac{1}{\mu_{1}} \sup _{s \in[0, t]}\left(\chi_{2}^{2}(s)\right) .
\end{aligned}
$$


As the boundedness of the extra term $e^{-\mu_{1} t} \int_{0}^{t} \chi_{2}^{2} e^{\mu_{1} \tau} d \tau$ is obtained from (32), directly applying Lemma 8 , we get the conclusion that signals $V_{1}(t), \varsigma_{1}(t)$, and $\int_{0}^{t} g_{1} N\left(\varsigma_{1}\right) \dot{\varsigma}_{1} d \tau$ hence $\chi_{1}, N\left(\varsigma_{1}\right), \widehat{\vartheta}_{1}$, and $\widetilde{\vartheta}_{1}$ are all bounded on $\left[0, t_{f}\right)$. Consequently, if $\chi_{2}$ is bounded, we can get the conclusion that all signals in Step 1 are bounded. In addition, the boundedness of $\chi_{2}$ will be proved in step 2 (see Step $k$ ).

Step $k(2 \leq k \leq n-1)$. Considering that steps $2 \leq k \leq n-1$ have a similar procedure, Step $k$ is presented as follows.

Choose the following Lyapunov-Krasovskii function:

$$
V_{k}=\frac{1}{2} \chi_{k}^{2}+\frac{1}{2 \gamma_{k}} \widetilde{\vartheta}_{k}^{2}+V_{k, 0}
$$

with $\gamma_{k}$ being a design positive parameter,

$$
\begin{aligned}
V_{k, 0}=\frac{e^{-r\left(t-\tau_{0}\right)}}{1-\tau^{*}}\left\{\sum_{i=2}^{k} \sum_{j=1}^{i} \int_{t-\tau_{j}(t)}^{t} e^{r s} H_{i, j}^{2}\left(x_{j}(s)\right) d s\right. \\
\left.+\frac{1}{2} \int_{t-\tau_{1}(t)}^{t} e^{r s} H_{1,1}^{2}\left(x_{1}(s)\right) d s\right\} .
\end{aligned}
$$

Similar to Step 1, the virtual control law $\alpha_{k}$ and adaptive laws are designed as

$$
\begin{gathered}
\alpha_{k}=\frac{q_{k}\left(\chi_{k}\right)}{2 a_{k}^{2}} N\left(\varsigma_{k}\right) \chi_{k} \widehat{\vartheta}_{k}, \\
\dot{\varsigma}_{k}=\frac{q_{k}\left(\chi_{k}\right)}{2 a_{k}^{2}} \chi_{k}^{2} \widehat{\vartheta}_{k}, \\
\dot{\widehat{\vartheta}}_{k}=\frac{\gamma_{k}}{2 a_{k}^{2}} \chi_{k}^{2}-\eta_{k} \widehat{\vartheta}_{k},
\end{gathered}
$$

with $a_{k}$ and $\eta_{k}$ being design positive parameters; the function $q_{k}\left(\chi_{k}\right)$ is defined as

$$
q_{k}\left(\chi_{k}\right)= \begin{cases}1, & \chi_{k} \notin \Omega_{\kappa_{k}} \\ 0, & \chi_{k} \in \Omega_{\kappa_{k}}\end{cases}
$$

where $\Omega_{\kappa_{k}}:=\left\{\chi_{k}|| \chi_{k} \mid<0.2554 \kappa_{k}\right\}$ stands for a compact set and $\kappa_{k}$ is a design positive parameter which decides the size of convergence region.

Case 1. In this case, we suppose that $\left|\chi_{k}\right|<0.2554 \kappa_{k}$. It is obvious that the tracking error $\chi_{k}$ is bounded. From (35)(37), when selecting bounded initial values, we achieve the boundedness of $\alpha_{k}, \varsigma_{k}$, and $N\left(\varsigma_{k}\right)$. After integrating (37) over $[0, t]$, we conclude that signal $\widehat{\vartheta}_{k}$ is bounded.

Case 2. In case 2, the tracking error satisfies $\left|\chi_{k}\right| \geq 0.2554 \kappa_{k}$.
The time derivative of $V_{k, 0}$ is

$$
\begin{aligned}
& \dot{V}_{k, 0} \\
& =-r V_{k, 0}+\frac{e^{-r\left(t-\tau_{0}\right)}}{1-\tau^{*}} \\
& \quad \times\left\{\sum_{i=2 j=1}^{k} \sum_{j}^{i}\left(e^{r t} H_{i, j}^{2}\left(x_{j}\right)-\left(1-\dot{\tau}_{j}\right) e^{r\left(t-\tau_{j}(t)\right)} H_{i, j}^{2}\left(x_{j}\left(\tau_{j}\right)\right)\right)\right. \\
& \left.\quad+\frac{1}{2} e^{r t} H_{1,1}^{2}\left(x_{1}\right)-\frac{1}{2}\left(1-\dot{\tau}_{j}\right) e^{r\left(t-\tau_{j}(t)\right)} H_{1,1}^{2}\left(x_{1}\left(\tau_{1}\right)\right)\right\} \\
& \leq-r V_{k, 0}+H_{k}\left(\overline{\mathbf{x}}_{k}\right)-\sum_{i=2}^{k} \sum_{j=1}^{i} H_{i, j}^{2}\left(x_{j}\left(\tau_{j}\right)\right)
\end{aligned}
$$$$
-\frac{1}{2} H_{1,1}^{2}\left(x_{1}\left(\tau_{1}\right)\right) \text {, }
$$

where $H_{k}\left(\overline{\mathbf{x}}_{k}\right)=\left(e^{r \tau_{0}} /\left(1-\tau^{*}\right)\right)\left\{\sum_{i=2}^{k} \sum_{j=1}^{i} H_{i, j}^{2}\left(x_{j}\right)+\right.$ $\left.(1 / 2) H_{1,1}^{2}\left(x_{1}\right)\right\}$.

Then the time derivative of $\chi_{k}$ is

$$
\dot{\chi}_{k}=f_{k}+g_{k} x_{k+1}+h_{k}\left(x_{1}\left(\tau_{1}\right), \ldots, x_{k}\left(\tau_{k}\right)\right)+d_{k}-\dot{\alpha}_{k-1},
$$

with

$$
\begin{aligned}
& \dot{\alpha}_{k-1} \\
& =\sum_{i=1}^{k-1}\left[\frac{\partial \alpha_{k-1}}{\partial c_{i}}\left(\frac{1}{2 a_{i}^{2}} e_{i}^{2} \widehat{\vartheta}_{i}\right)\right. \\
& +\frac{\partial \alpha_{k-1}}{\partial x_{i}}\left(f_{i}+g_{i} x_{i+1}+h_{i}\left(x_{1}\left(\tau_{1}\right), \ldots, x_{i}\left(\tau_{i}\right)\right)\right. \\
& \left.\left.+d_{i}(t)\right)+\frac{\partial \alpha_{k-1}}{\partial \widehat{\vartheta}_{i}}\left(\frac{\gamma_{i}}{2 a_{i}^{2}} e_{i}^{2}-\gamma_{i} \widehat{\vartheta}_{i}\right)\right] \\
& +\frac{\partial \alpha_{k-1}}{\partial y_{d}} \dot{y}_{d}
\end{aligned}
$$

Remark 10. Here $\alpha_{k-1}$ is the function of $\alpha_{k-2}$. When $k=2$, we have $\alpha_{k-2}=y_{d}$; that is, $\alpha_{k-1}$ is the function of $\mathbf{x}_{k-1}$, $y_{d}, \boldsymbol{\varsigma}_{k-1}$, and $\overline{\widehat{\vartheta}}_{k-1}$, where $\boldsymbol{\varsigma}_{k-1}=\left[\varsigma_{1}, \ldots, \varsigma_{k-1}\right]^{T}$ and $\overline{\widehat{\vartheta}}_{k-1}=$ $\left[\widehat{\vartheta}_{1}, \ldots, \widehat{\vartheta}_{k-1}\right]^{T}$. Then we derive the time derivative of $\alpha_{k-1}$ in (41).

Using (40) and (41), the derivative of $V_{k}$ is

$$
\begin{aligned}
\dot{V}_{k}=\chi_{k}\left(f_{k}+g_{k} x_{k+1}+h_{k}\left(x_{1}\left(\tau_{1}\right), \ldots, x_{k}\left(\tau_{k}\right)\right)\right. \\
\left.+d_{k}-\dot{\alpha}_{k-1}\right)-\frac{1}{\gamma_{k}} \widetilde{\vartheta}_{k} \dot{\hat{\vartheta}}_{k}+\dot{V}_{k, 0} \\
=\chi_{k}\left\{f_{k}+g_{k} x_{k+1}+h_{k}\left(x_{1}\left(\tau_{1}\right), \ldots, x_{k}\left(\tau_{k}\right)\right)\right.
\end{aligned}
$$




$$
\begin{aligned}
& +d_{k}-\sum_{i=1}^{k-1}\left[\frac{\partial \alpha_{k-1}}{\partial \varsigma_{i}} \dot{\zeta}_{i}+\frac{\partial \alpha_{k-1}}{\partial x_{i}} \dot{x}_{i}+\frac{\partial \alpha_{k-1}}{\partial \widehat{\vartheta}_{i}} \dot{\hat{\vartheta}}_{i}\right] \\
& \left.-\frac{\partial \alpha_{k-1}}{\partial y_{d}} \dot{y}_{d}\right\}-\frac{1}{\gamma_{k}} \widetilde{\vartheta}_{k} \dot{\hat{\vartheta}}_{k}+\dot{V}_{k, 0} .
\end{aligned}
$$

Owing to Assumption 3, we get

$$
\begin{array}{r}
\left|h_{i}\left(x_{1}\left(\tau_{1}\right), \ldots, x_{i}\left(\tau_{i}\right)\right)\right|^{2} \leq 2 \sum_{j=1}^{i} H_{i, j}^{2}\left(x_{j}\left(\tau_{j}\right)\right), \\
2 \leq i \leq n .
\end{array}
$$

Utilizing Young's inequality (42) yields

$$
\begin{aligned}
\dot{V}_{k} \leq & -c_{k} \chi_{k}^{2}+\chi_{k} g_{k} \alpha_{k}+\chi_{k+1}^{2} \\
+ & \chi_{k}\left\{f_{k}-\sum_{i=1}^{k-1}\left[\frac{\partial \alpha_{k-1}}{\partial \varsigma_{i}}\left(\frac{1}{2 a_{i}^{2}} \chi_{i}^{2} \widehat{\vartheta}_{i}\right)+\frac{\partial \alpha_{k-1}}{\partial x_{i}}\left(f_{i}+g_{i} x_{i+1}\right)\right.\right. \\
& \left.+\frac{\partial \alpha_{k-1}}{\partial \widehat{\vartheta}_{i}}\left(\frac{\gamma_{i}}{2 a_{i}^{2}} \chi_{i}^{2}-\gamma_{i} \widehat{\vartheta}_{i}\right)\right]-\frac{\partial \alpha_{k-1}}{\partial y_{d}} \dot{y}_{d} \\
& +\left(\frac{1}{2 \rho^{2}}+\frac{1}{2}\right) \sum_{i=1}^{k-1}\left(\frac{\partial \alpha_{k-1}}{\partial x_{i}}\right)^{2} \chi_{k} \\
& \left.+\left(\frac{1}{2 \rho^{2}}+c_{k}+\frac{1}{4} g_{k}^{2}+1\right) \chi_{k}\right\} \\
-\frac{1}{2} \chi_{k}^{2} & +\sum_{i=2}^{k} \sum_{j=1}^{i} H_{i, j}^{2}\left(x_{j}\left(\tau_{j}\right)\right) \\
+ & \frac{1}{2} H_{1,1}^{2}\left(x_{1}\left(\tau_{1}\right)\right)+\frac{1}{2} \sum_{i=1}^{k} \rho^{2} \bar{d}_{i}^{2}-\frac{1}{\gamma_{k}} \widetilde{\vartheta}_{k} \dot{\hat{\vartheta}}_{k}+\dot{V}_{k, 0} .
\end{aligned}
$$

Substituting (39) into (44) results in

$$
\begin{aligned}
\dot{V}_{k} \leq & -c_{k} \chi_{k}^{2}-r V_{k, 0}+\chi_{k} g_{k} \alpha_{k}+\chi_{k+1}^{2} \\
& +\chi_{k} \bar{f}_{k}-\frac{1}{2} \chi_{k}^{2}+\frac{1}{2} \sum_{i=1}^{k} \rho^{2} \bar{d}_{i}^{2}-\frac{1}{\gamma_{k}} \widetilde{\vartheta}_{k} \dot{\hat{\vartheta}}_{k} \\
& +\left(1-16 \tanh ^{2}\left(\frac{\chi_{k}}{\kappa_{k}}\right)\right) H_{k}\left(\overline{\mathbf{x}}_{k}\right),
\end{aligned}
$$

with

$$
\begin{gathered}
\overline{f_{k}}=f_{k}-\sum_{i=1}^{k-1}\left[\frac{\partial \alpha_{k-1}}{\partial \varsigma_{i}}\left(\frac{1}{2 a_{i}^{2}} \chi_{i}^{2} \widehat{\vartheta}_{i}\right)+\frac{\partial \alpha_{k-1}}{\partial x_{i}}\left(f_{i}+g_{i} x_{i+1}\right)\right. \\
\left.+\frac{\partial \alpha_{k-1}}{\partial \widehat{\vartheta}_{i}}\left(\frac{\gamma_{i}}{2 a_{i}^{2}} \chi_{i}^{2}-\gamma_{i} \widehat{\vartheta}_{i}\right)\right]-\frac{\partial \alpha_{k-1}}{\partial y_{d}} \dot{y}_{d} \\
+\left(\frac{1}{2 \rho^{2}}+\frac{1}{2}\right) \sum_{i=1}^{k-1}\left(\frac{\partial \alpha_{k-1}}{\partial x_{i}}\right)^{2} \chi_{k}
\end{gathered}
$$

$$
\begin{aligned}
& +\left(\frac{1}{2 \rho^{2}}+c_{k}+\frac{1}{4} g_{k}^{2}+1\right) \chi_{k} \\
& +\frac{16}{\chi_{k}} \tanh ^{2}\left(\frac{\chi_{k}}{\kappa_{k}}\right) H_{k}\left(\overline{\mathbf{x}}_{k}\right) .
\end{aligned}
$$

Similarly, $\overline{f_{k}}$ can be approximated by FLSs to an arbitrary given accuracy as

$$
\overline{f_{k}}=\boldsymbol{\theta}_{k}^{* T} \boldsymbol{\xi}_{k}\left(\mathbf{Z}_{k}\right)+\delta_{k}\left(\mathbf{Z}_{k}\right), \quad\left|\delta_{k}\left(\mathbf{Z}_{k}\right)\right| \leq \varepsilon_{k},
$$

where $\mathbf{Z}_{k}=\left[\mathbf{x}_{k}, y_{d}, \dot{y}_{d}, \boldsymbol{\varsigma}_{k-1}, \overline{\widehat{\vartheta}}_{k-1}\right]^{T}, \delta_{k}\left(\mathbf{Z}_{k}\right)$ represents approximation error, and $\varepsilon_{k}$ is an unknown positive constant.

As the fuzzy basis function $\boldsymbol{\xi}_{k}\left(\mathbf{Z}_{k}\right)$ satisfies $0<$ $\boldsymbol{\xi}_{k}^{T}\left(\mathbf{Z}_{k}\right) \boldsymbol{\xi}_{k}\left(\mathbf{Z}_{k}\right) \leq 1$, we get the following inequality:

$$
\begin{aligned}
\chi_{k} \overline{f_{k}} & =\chi_{k} \boldsymbol{\theta}_{k}^{* T} \boldsymbol{\xi}_{k}\left(\mathbf{Z}_{k}\right)+\chi_{k} \delta_{k}\left(\mathbf{Z}_{k}\right) \\
& \leq \frac{1}{2 a_{k}^{2}} \chi_{k}^{2} \vartheta_{k}+\frac{1}{2} a_{k}^{2}+\frac{1}{2} \chi_{k}^{2}+\frac{1}{2} \varepsilon_{k}^{2} .
\end{aligned}
$$

Substituting (48) into (45), we obtain

$$
\begin{aligned}
\dot{V}_{k} \leq & -c_{k} \chi_{k}^{2}-r V_{k, 0}+\chi_{k} g_{k} \alpha_{k}+\chi_{k+1}^{2}-\frac{1}{\gamma_{k}} \widetilde{\vartheta}_{k} \dot{\hat{\vartheta}}_{k} \\
& +\frac{1}{2 a_{k}^{2}} \chi_{k}^{2} \vartheta_{k}+\frac{1}{2} a_{k}^{2}+\frac{1}{2} \varepsilon_{k}^{2}+\frac{1}{2} \sum_{i=1}^{k} \rho^{2} \bar{d}_{i}^{2} \\
& +\left(1-16 \tanh ^{2}\left(\frac{\chi_{k}}{\kappa_{k}}\right)\right) H_{k}\left(\overline{\mathbf{x}}_{k}\right) .
\end{aligned}
$$

As $\left|\chi_{k}\right| \geq 0.2554 \kappa_{k}$, we can derive

$$
\left(1-16 \tanh ^{2}\left(\frac{\chi_{k}}{\kappa_{k}}\right)\right) H_{k}\left(\overline{\mathbf{x}}_{k}\right) \leq 0 .
$$

Applying (35)-(37) and (50), (49) produces

$$
\dot{V}_{k} \leq-\mu_{k} V_{k}+\chi_{k+1}^{2}+\left[g_{k} N\left(\varsigma_{k}\right)+1\right] \dot{\varsigma}_{k}+C_{k},
$$

where $\mu_{k}=\min \left(2 c_{k}, r, \eta_{k}\right)$ and $C_{k}=(1 / 2) a_{k}^{2}+(1 / 2) \varepsilon_{k}^{2}+$ $(1 / 2) \sum_{i=1}^{k} \rho^{2} \bar{d}_{i}^{2}+\left(\eta_{k} / 2 \gamma_{k}\right) \vartheta_{k}^{2}$.

Multiplying (51) by $e^{\mu_{k} t}$ results in

$$
\frac{d}{d t}\left(V_{k} e^{\mu_{k} t}\right) \leq e^{\mu_{k} t} \chi_{k+1}^{2}+\left[g_{k} N\left(\varsigma_{k}\right)+1\right] \dot{\zeta}_{k} e^{\mu_{k} t}+C_{k} e^{\mu_{k} t} .
$$

Integrating $(52)$ over $[0, t]$, we obtain

$$
\begin{aligned}
V_{k} \leq & \beta_{k}+e^{-\mu_{k} t} \int_{0}^{t}\left[g_{k} N\left(\varsigma_{k}\right)+1\right] \dot{\xi}_{k} e^{\mu_{k} \tau} d \tau \\
& +e^{-\mu_{k} t} \int_{0}^{t} \chi_{k+1}^{2} e^{\mu_{k} \tau} d \tau,
\end{aligned}
$$

with $\beta_{k}=\left(C_{k} / \mu_{k}\right)+\left(V_{k}(0)-\left(C_{k} / \mu_{k}\right)\right) e^{-\mu_{k} t}$. 
Remark 11. The discussion of (53) is similar to the analysis of (31). If $\chi_{k+1}$ can be regulated as bounded, by utilizing Lemma 8 , the boundedness of signals $V_{k}(t), \varsigma_{k}(t)$, and $\int_{0}^{t} g_{k} N\left(\varsigma_{k}\right) \dot{\zeta}_{k} d \tau$ is achieved. Thus, we can guarantee that signals $\chi_{k}, N\left(\varsigma_{k}\right), \widehat{\vartheta}_{k}$, and $\widetilde{\vartheta}_{k}$ are all bounded on $\left[0, t_{f}\right)$. The effect of the extra term $e^{-\mu_{k} t} \int_{0}^{t} e_{k+1}^{2} e^{\mu_{k} \tau} d \tau$ will be handled in the next step.

Step $n$. Consider Lyapunov-Krasovskii function as follows:

$$
V_{n}=\frac{1}{2} \chi_{n}^{2}+\frac{1}{2 \gamma_{n}} \widetilde{\vartheta}_{n}^{2}+V_{n, 0}
$$

where $\gamma_{n}$ is a design positive parameter, and

$$
\begin{aligned}
V_{n, 0}=\frac{e^{-r\left(t-\tau_{0}\right)}}{1-\tau^{*}}\left\{\sum_{i=2}^{n} \sum_{j=1}^{i} \int_{t-\tau_{j}(t)}^{t} e^{r s} H_{i, j}^{2}\left(x_{j}(s)\right) d s\right. \\
\left.+\frac{1}{2} \int_{t-\tau_{1}(t)}^{t} e^{r s} H_{1,1}^{2}\left(x_{1}(s)\right) d s\right\} .
\end{aligned}
$$

We choose the following actual control input $u$ and adaptive laws:

$$
\begin{gathered}
u=\frac{q_{n}\left(\chi_{n}\right)}{2 a_{n}^{2}} N\left(s_{n}\right) \chi_{n} \widehat{\vartheta}_{n}, \\
\dot{\zeta}_{n}=\frac{q_{n}\left(\chi_{n}\right)}{2 a_{n}^{2}} \chi_{n}^{2} \widehat{\vartheta}_{n}, \\
\dot{\widehat{\vartheta}}_{n}=\frac{\gamma_{n}}{2 a_{n}^{2}} \chi_{n}^{2}-\eta_{n} \widehat{\vartheta}_{n},
\end{gathered}
$$

where $a_{n}$ and $\eta_{n}$ are design positive parameters. The function $q_{n}\left(\chi_{n}\right)$ is defined as

$$
q_{n}\left(\chi_{n}\right)= \begin{cases}1, & \chi_{n} \notin \Omega_{\kappa_{n}}, \\ 0, & \chi_{n} \in \Omega_{\kappa_{n}},\end{cases}
$$

with $\Omega_{\kappa_{n}}:=\left\{\chi_{n}|| \chi_{n} \mid<0.2554 \kappa_{n}\right\}$ denoting a compact set and $\kappa_{n}$ is a design positive parameter.

Similarly, we analyze the $n$ th-subsystem from two cases.

Case 1. In Case $1, \chi_{n}$ satisfies $\left|\chi_{n}\right|<0.2554 \kappa_{n}$. As $\kappa_{n}$ is a positive design parameter; we obtain that $\chi_{n}$ is bounded. In addition, we can conclude that the signals $u, \varsigma_{n}, N\left(\varsigma_{n}\right), \widehat{\vartheta}_{n}$, and $\widetilde{\vartheta}_{n}$ are bounded.

Case 2. We suppose that $\left|\chi_{n}\right| \geq 0.2554 \kappa_{n}$ in this case.

The time derivative of $V_{n}$ is

$$
\begin{gathered}
\dot{V}_{n}=\chi_{n}\left(f_{n}+g_{n} \boldsymbol{\eta}^{T} \boldsymbol{\kappa} u+g_{n} d(u)+h_{n}\left(x_{1}\left(\tau_{1}\right), \ldots, x_{n}\left(\tau_{n}\right)\right)\right. \\
\left.+d_{n}-\dot{\alpha}_{n-1}\right)-\frac{1}{\gamma_{n}} \widetilde{\vartheta}_{n} \dot{\widehat{\vartheta}}_{n}+\dot{V}_{n, 0} .
\end{gathered}
$$

From the definition of $\alpha_{n-1}$, we obtain

$\dot{\alpha}_{n-1}$

$$
\begin{aligned}
=\sum_{i=1}^{n-1}[ & \frac{\partial \alpha_{n-1}}{\partial \varsigma_{i}}\left(\frac{1}{2 a_{i}^{2}} \chi_{i}^{2} \widehat{\vartheta}_{i}\right) \\
& +\frac{\partial \alpha_{n-1}}{\partial x_{i}}\left(f_{i}+g_{i} x_{i+1}+h_{i}\left(x_{1}\left(\tau_{1}\right), \ldots, x_{i}\left(\tau_{i}\right)\right)+d_{i}\right) \\
& \left.+\frac{\partial \alpha_{n-1}}{\partial \widehat{\vartheta}_{i}}\left(\frac{\gamma_{i}}{2 a_{i}^{2}} \chi_{i}^{2}-\gamma_{i} \widehat{\vartheta}_{i}\right)\right]+\frac{\partial \alpha_{n-1}}{\partial y_{d}} \dot{y}_{d}
\end{aligned}
$$

Applying Young's inequality and (43), (60) can be rewritten as

$$
\begin{aligned}
& \dot{V}_{n} \\
& \leq-c_{n} \chi_{n}^{2}+\chi_{n} g_{n} \boldsymbol{\eta}^{T} \boldsymbol{\kappa} u \\
& +\chi_{n}\left\{f_{n}-\sum_{i=1}^{n-1}\left[\frac{\partial \alpha_{n-1}}{\partial \varsigma_{i}}\left(\frac{1}{2 a_{i}^{2}} \chi_{i}^{2} \widehat{\vartheta}_{i}\right)+\frac{\partial \alpha_{n-1}}{\partial x_{i}}\left(f_{i}+g_{i} x_{i+1}\right)\right.\right. \\
& \left.+\frac{\partial \alpha_{n-1}}{\partial \widehat{\vartheta}_{i}}\left(\frac{\gamma_{i}}{2 a_{i}^{2}} \chi_{i}^{2}-\gamma_{i} \widehat{\vartheta}_{i}\right)\right]-\frac{\partial \alpha_{n-1}}{\partial y_{d}} \dot{y}_{d} \\
& +\left(\frac{1}{2 \rho^{2}}+\frac{1}{2}\right) \sum_{i=1}^{n-1}\left(\frac{\partial \alpha_{n-1}}{\partial x_{i}}\right)^{2} \chi_{n} \\
& \left.+\left(\frac{1}{2 \rho^{2}}+c_{n}+1\right) \chi_{n}\right\}-\frac{1}{2} \chi_{n}^{2}+\sum_{i=2}^{n} \sum_{j=1}^{i} H_{i, j}^{2}\left(x_{j}\left(\tau_{j}\right)\right) \\
& +\frac{1}{2} H_{1,1}^{2}\left(x_{1}\left(\tau_{1}\right)\right)+\frac{1}{2} \sum_{i=1}^{n} \rho^{2} \bar{d}_{i}^{2}-\frac{1}{\gamma_{n}} \widetilde{\vartheta}_{n} \dot{\widehat{\vartheta}}_{n}+\dot{V}_{n, 0} .
\end{aligned}
$$

The time derivative of $V_{n, 0}$ is

$\dot{V}_{n, 0}$

$$
\begin{aligned}
= & -r V_{n, 0}+\frac{e^{-r\left(t-\tau_{0}\right)}}{1-\tau^{*}} \\
& \times\left\{\frac{1}{2} e^{r t} H_{1,1}^{2}\left(x_{1}\right)-\frac{1}{2}\left(1-\dot{\tau}_{1}\right) e^{r\left(t-\tau_{1}(t)\right)} H_{1,1}^{2}\left(x_{1}\left(\tau_{1}\right)\right)\right. \\
& \left.+\sum_{i=2}^{n} \sum_{j=1}^{i}\left(e^{r t} H_{i, j}^{2}\left(x_{j}\right)-\left(1-\dot{\tau}_{j}\right) e^{r\left(t-\tau_{j}(t)\right)} H_{i, j}^{2}\left(x_{j}\left(\tau_{j}\right)\right)\right)\right\} \\
\leq & -r V_{n, 0}+H_{n}\left(\overline{\mathbf{x}}_{n}\right)-\sum_{i=2}^{n} \sum_{j=1}^{i} H_{i, j}^{2}\left(x_{j}\left(\tau_{j}\right)\right) \\
& -\frac{1}{2} H_{1,1}^{2}\left(x_{1}\left(\tau_{1}\right)\right),
\end{aligned}
$$


where $H_{n}\left(\overline{\mathbf{x}}_{n}\right)=\left(e^{r \tau_{0}} /\left(1-\tau^{*}\right)\right)\left\{\sum_{i=2}^{n} \sum_{j=1}^{i} H_{i, j}^{2}\left(x_{j}\right)+(1 / 2) H_{1,1}^{2}\right.$ $\left.\left(x_{1}\right)\right\}$.

By utilizing (63), (62) yields

$$
\begin{aligned}
\dot{V}_{n} \leq & -c_{n} \chi_{n}^{2}-r V_{n, 0}+\chi_{n} g_{n} \boldsymbol{\eta}^{T} \boldsymbol{\kappa} u+\chi_{n} \overline{f_{n}} \\
& -\frac{1}{2} \chi_{n}^{2}+\frac{1}{2} \sum_{i=1}^{n} \rho^{2} \bar{d}_{i}^{2}-\frac{1}{\gamma_{n}} \widetilde{\vartheta}_{n} \dot{\hat{\vartheta}}_{n} \\
& +\left(1-16 \tanh ^{2}\left(\frac{\chi_{n}}{\kappa_{n}}\right)\right) H_{n}\left(\overline{\mathbf{x}}_{n}\right),
\end{aligned}
$$

with

$$
\begin{aligned}
\overline{f_{n}}=f_{n}-\sum_{i=1}^{n-1}\left[\frac{\partial \alpha_{n-1}}{\partial \varsigma_{i}}\left(\frac{1}{2 a_{i}^{2}} \chi_{i}^{2} \widehat{\vartheta}_{i}\right)+\frac{\partial \alpha_{n-1}}{\partial x_{i}}\left(f_{i}+g_{i} x_{i+1}\right)\right. \\
\left.+\frac{\partial \alpha_{n-1}}{\partial \widehat{\vartheta}_{i}}\left(\frac{\gamma_{i}}{2 a_{i}^{2}} \chi_{i}^{2}-\gamma_{i} \widehat{\vartheta}_{i}\right)\right]-\frac{\partial \alpha_{n-1}}{\partial y_{d}} \dot{y}_{d} \\
+\left(\frac{1}{2 \rho^{2}}+\frac{1}{2}\right) \sum_{i=1}^{n-1}\left(\frac{\partial \alpha_{n-1}}{\partial x_{i}}\right)^{2} \chi_{n}+\left(\frac{1}{2 \rho^{2}}+c_{n}+1\right) \chi_{n} \\
+\frac{16}{\chi_{n}} \tanh ^{2}\left(\frac{\chi_{n}}{\kappa_{n}}\right) H_{n}\left(\overline{\mathbf{x}}_{n}\right) .
\end{aligned}
$$

By using FLSs, function $\overline{f_{n}}$ can be approximated as

$$
\overline{f_{n}}=\boldsymbol{\theta}_{n}^{* T} \boldsymbol{\xi}_{n}\left(\mathbf{Z}_{n}\right)+\delta_{n}\left(\mathbf{Z}_{n}\right), \quad\left|\delta_{n}\left(\mathbf{Z}_{n}\right)\right| \leq \varepsilon_{n},
$$

where $\mathbf{Z}_{n}=\left[\overline{\mathbf{x}}_{n}, y_{d}, \dot{y}_{d}, \boldsymbol{\varsigma}_{n-1}, \overline{\widehat{\vartheta}}_{n-1}\right]^{T}$ with $\boldsymbol{\varsigma}_{n-1}=\left[\varsigma_{1}, \ldots, \varsigma_{n-1}\right]^{T}$ and $\overline{\widehat{\vartheta}}_{n-1}=\left[\widehat{\vartheta}_{1}, \ldots, \widehat{\vartheta}_{n-1}\right]^{T}, \delta_{n}\left(\mathbf{Z}_{n}\right)$ expresses the approximation error, and $\varepsilon_{n}$ is an unknown positive constant.

Similarly, we can derive the following inequality:

$$
\begin{aligned}
\chi_{n} \overline{f_{n}} & =\chi_{n} \boldsymbol{\theta}_{n}^{* T} \boldsymbol{\xi}_{n}\left(\mathbf{Z}_{n}\right)+\chi_{n} \delta_{n}\left(\mathbf{Z}_{n}\right) \\
& \leq \frac{1}{2 a_{n}^{2}} \chi_{n}^{2} \vartheta_{n}+\frac{1}{2} a_{n}^{2}+\frac{1}{2} \chi_{n}^{2}+\frac{1}{2} \varepsilon_{n}^{2} .
\end{aligned}
$$

Considering $\left|\chi_{n}\right| \geq 0.2554 \kappa_{n}$, we get

$$
\left(1-16 \tanh ^{2}\left(\frac{\chi_{n}}{\kappa_{n}}\right)\right) H_{n}\left(\overline{\mathbf{x}}_{n}\right) \leq 0 .
$$

Applying (56)-(58), (67), and (68), (64) results in

$$
\dot{V}_{n} \leq-\mu_{n} V_{n}+\left[g_{n} \boldsymbol{\eta}^{T} \boldsymbol{\kappa} N\left(\varsigma_{n}\right)+1\right] \dot{\varsigma}_{n}+C_{n},
$$

where $C_{n}=(1 / 2) a_{n}^{2}+(1 / 2) \varepsilon_{n}^{2}+(1 / 2) \sum_{i=1}^{n} \rho^{2} \bar{d}_{i}^{2}+(1 / 2) \rho^{2} d_{0}^{2}+$ $\left(\eta_{n} / 2 \gamma_{n}\right) \vartheta_{n}^{2}$ and $\mu_{n}=\min \left(2 c_{n}, r, \eta_{n}\right)$.

Multiplying (69) by $e^{\mu_{n} t}$ and then integrating it over $[0, t]$, we have

$$
V_{n} \leq \beta_{n}+e^{-\mu_{n} t} \int_{0}^{t}\left[g_{n} \boldsymbol{\eta}^{T} \boldsymbol{\kappa} N\left(\varsigma_{n}\right)+1\right] \dot{\varsigma}_{n} e^{\mu_{n} \tau} d \tau,
$$

with $\beta_{n}=\left(C_{n} / \mu_{n}\right)+\left(V_{n}(0)-\left(C_{n} / \mu_{n}\right)\right) e^{-\mu_{n} t}$.
Considering function $\boldsymbol{\eta}^{T}(t) \boldsymbol{\kappa}(t)$ satisfies $\boldsymbol{\eta}^{T}(t) \boldsymbol{\kappa}(t) \quad \epsilon$ $\left[\min \left(\varphi_{r 0}, \varphi_{l 0}\right), \varphi_{r 1}+\varphi_{l 1}\right]$, and Assumption 2 holds, we can derive $\left|g_{n} \boldsymbol{\eta}^{T}(t) \boldsymbol{\kappa}(t)\right| \in\left[g_{n 0} \min \left(\varphi_{r 0}, \varphi_{l 0}\right),\left(\varphi_{r 1}+\varphi_{l 1}\right) g_{n 1}\right]$. Noting (70), applying Lemma 8 , we can conclude that signals $V_{n}(t), \varsigma_{n}(t)$, and $\int_{0}^{t} g_{n} \boldsymbol{\eta}^{T} \boldsymbol{\kappa} N\left(\varsigma_{n}\right) \dot{\varsigma}_{n} d \tau$ are bounded. Hence, $\chi_{n}$, $u, N\left(\varsigma_{n}\right), \widehat{\vartheta}_{n}$, and $\widetilde{\vartheta}_{n}$ are SUUB on $\left[0, t_{f}\right)$.

The main result is summarized in the following theorem.

Theorem 12. Consider nonaffine nonlinear time-varying delay system (1), when Assumptions 1-6 hold, by applying the control law (56), virtual control laws (19), and (35) and adaptive laws (20), (21), (36), (37), (57), and (58); then with bounded initial conditions, it is guaranteed that all the signals in closed-loop system are SUUB and the tracking error eventually converges to a small neighbourhood of the origin.

Proof. Owing to the previous analysis, we get the conclusion that the term $\int_{0}^{t}\left[g_{n} \boldsymbol{\eta}^{T} \boldsymbol{\kappa} N\left(\varsigma_{n}\right)+1\right] \dot{\zeta}_{n} d \tau$ is bounded.

Noting (70), we suppose that the upper bound $\lambda_{n}$ satisfies

$$
\int_{0}^{t}\left|\left[g_{n} \boldsymbol{\eta}^{T} \boldsymbol{\kappa} N\left(\varsigma_{n}\right)+1\right] \dot{\varsigma}_{n}\right| e^{\mu_{n}(\tau-t)} d \tau \leq \lambda_{n}
$$

From (54), (70), and (71), we have

$$
\begin{gathered}
\left|\chi_{n}\right| \leq \sqrt{2\left(\beta_{n}+\lambda_{n}\right)}, \\
\left|\widetilde{\vartheta}_{n}\right| \leq \sqrt{2 \gamma_{n}\left(\beta_{n}+\lambda_{n}\right)} .
\end{gathered}
$$

Thus, we can conclude the boundedness of the signals $\chi_{n}$, $\widehat{\vartheta}_{n}$, and $\widetilde{\vartheta}_{n}$.

In the rest of the steps from $n-1$ to 1 , we acquire

$$
\begin{aligned}
V_{i} \leq & \beta_{i}+e^{-\mu_{i} t} \int_{0}^{t}\left[g_{i} N\left(\varsigma_{i}\right)+1\right] \dot{\zeta}_{i} e^{\mu_{i} \tau} d \tau \\
& +e^{-\mu_{i} t} \int_{0}^{t} \chi_{i+1}^{2} e^{\mu_{i} \tau} d \tau, \quad i=1, \ldots, n-1 .
\end{aligned}
$$

As the boundedness of $\chi_{i+1}$ is guaranteed in step $i+1$, we define an upper bound $\lambda_{i}$ as

$$
\int_{0}^{t}\left|\left[g_{i} N\left(\varsigma_{i}\right)+1\right] \dot{\zeta}_{i}\right| e^{\mu_{i}(\tau-t)} d \tau+\int_{0}^{t} \chi_{i+1}^{2} e^{\mu_{i}(\tau-t)} d \tau \leq \lambda_{i} .
$$

Investigating the definition of $V_{i}(t)$ and combing (73) and (74), we obtain

$$
\begin{gathered}
\left|\chi_{i}\right| \leq \sqrt{2\left(\beta_{i}+\lambda_{i}\right)}, \\
\left|\widetilde{\vartheta}_{i}\right| \leq \sqrt{2 \gamma_{i}\left(\beta_{i}+\lambda_{i}\right)} .
\end{gathered}
$$

Hence, signals $\chi_{i}, \widehat{\vartheta}_{i}$, and $\widetilde{\vartheta}_{i}$ are bounded.

According to the whole abovementioned analysis, the boundedness of all signals in closed-loop system is proved. The tracking error $\chi_{1}$ converges to a small neighbourhood of the origin by selecting appropriate design parameters.

Remark 13. According to the above analysis, we know that tracking error depends on $a_{i}, \vartheta_{i}, \varepsilon_{i}, \lambda_{i}, \gamma_{i}, \eta_{i}, r, c_{i}$, and $\overline{d_{i}}$. As 
$\vartheta_{i}, \varepsilon_{i}, \lambda_{i}$, and $\overline{d_{i}}$ are unknown, a concrete estimation of the tracking error is impossible. From inequality (75), it is clear that by reducing $a_{i}$ and $\eta_{i}$, meanwhile increasing $c_{i}, \gamma_{i}$, and $r$, the tracking error will be diminished. Simultaneously, it is worth pointing out that the parameters $\vartheta_{i}, \varepsilon_{i}, \lambda_{i}$, and $\overline{d_{i}}$ are not used in the control law and adaptive laws design, which are employed for stability analysis.

\section{Simulation}

In this section, two simulation examples are employed to validate the effectiveness of the proposed adaptive fuzzy tracking control approach. The desired tracking trajectory is $y_{d}=\sin (t)+\cos (0.5 t)$. The dead-zone $D(u)$ is defined as

$$
D(u)= \begin{cases}(1-0.3 \sin (u))(u-0.5), & u>0.5 \\ 0, & -0.25 \leq u \leq 0.5 \\ (0.8-0.2 \cos (u))(u+0.25), & u<-0.25\end{cases}
$$

Example 1. Consider the following nonlinear time-delay system:

$$
\begin{gathered}
\dot{x}_{1}(t)=x_{1}^{2}(t)+x_{1}(t) x_{2}(t)+0.5 x_{1}\left(t-\tau_{1}(t)\right)+0.5 \cos (t), \\
\dot{x}_{2}(t)=-x_{1}(t)+\frac{D(u(t))}{\sqrt{|D(u(t))|+0.1}}-2 x_{1}^{2}(t) x_{2}(t), \\
+x_{1}^{2}\left(t-\tau_{1}(t)\right) x_{2}\left(t-\tau_{2}(t)\right)+0.2 \sin (t), \\
y=x_{1},
\end{gathered}
$$

where $\tau_{1}(t)=0.2(1+\cos (t))$ and $\tau_{2}(t)=0.3(1+\sin (t))$.

According to Theorem 12, the control laws and the adaptive laws are chosen as

$$
\begin{aligned}
\alpha_{1}=\frac{q_{1}\left(\chi_{1}\right)}{2 a_{1}^{2}} N\left(\varsigma_{1}\right) \chi_{1} \widehat{\vartheta}_{1}, & u=\frac{q_{2}\left(\chi_{2}\right)}{2 a_{2}^{2}} N\left(\varsigma_{2}\right) \chi_{2} \widehat{\vartheta}_{2}, \\
\dot{\varsigma}_{1}=\frac{q_{1}\left(\chi_{1}\right)}{2 a_{1}^{2}} \chi_{1}^{2} \widehat{\vartheta}_{1}, & \dot{\hat{\vartheta}}_{1}=\frac{\gamma_{1}}{2 a_{1}^{2}} \chi_{1}^{2}-\eta_{1} \widehat{\vartheta}_{1}, \\
\dot{\zeta}_{2}=\frac{q_{2}\left(\chi_{2}\right)}{2 a_{2}^{2}} \chi_{2}^{2} \widehat{\vartheta}_{2}, & \dot{\hat{\vartheta}}_{2}=\frac{\gamma_{2}}{2 a_{2}^{2}} \chi_{2}^{2}-\eta_{2} \widehat{\vartheta}_{2} .
\end{aligned}
$$

In the simulation, the design parameters are selected as $a_{1}=0.5, a_{2}=0.5, \gamma_{1}=10, \gamma_{2}=20, \eta_{1}=0.1, \eta_{2}=0.1$, $\kappa_{1}=0.1, \kappa_{2}=0.1$, and $r=1$. The initial values are chosen as $x_{1}(\omega)=0.1$ and $x_{2}(\omega)=0$ for $\omega \leq 0, \varsigma_{1}(0)=0.01, \varsigma_{2}(0)=$ $0.01, \widehat{\vartheta}_{1}(0)=0.01$, and $\widehat{\vartheta}_{2}(0)=0.01$. The simulation results are shown in Figures 1-5, respectively.

From Figure 1, it can be seen that good tracking performance is achieved. The response curve of state variable is shown in Figure 2. Figure 3 depicts the trajectory of the control input. We can conclude that the control input is bounded. Figures 4 and 5 display the adaptive parameters $\widehat{\vartheta}_{1}$, $\widehat{\vartheta}_{2}$, and $\varsigma_{1}, \varsigma_{2}$, respectively.

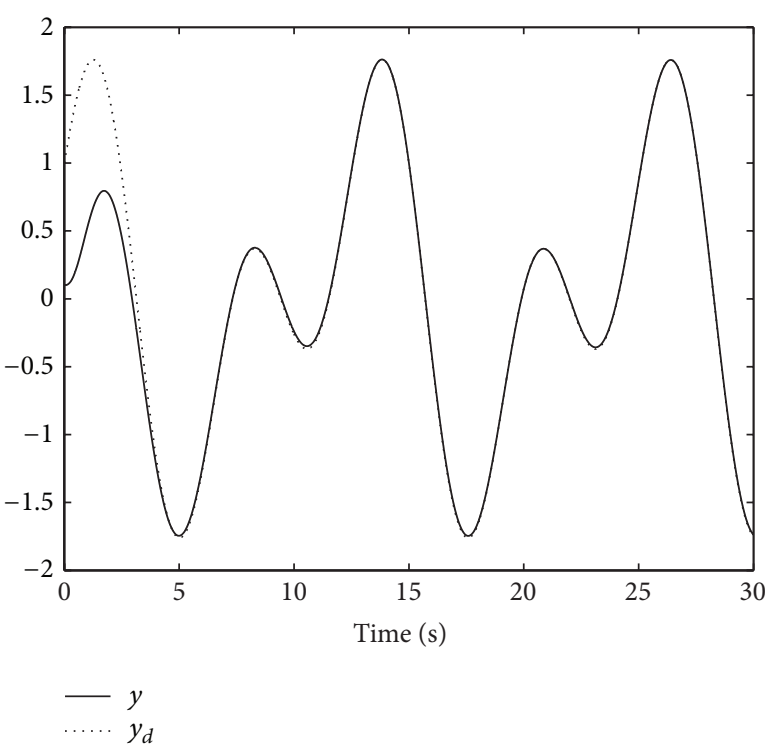

FIGURE 1: Trajectories of system output $y$ and reference signal $y_{d}$.

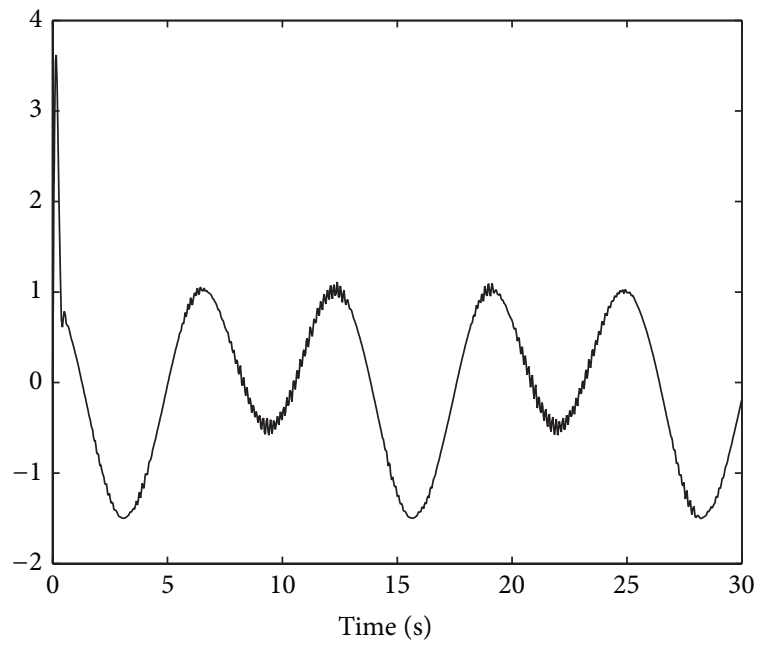

FIGURE 2: Trajectory of the system state $x_{2}$.

Example 2. To further demonstrate the feasibility of the controller, we present the following nonlinear system:

$$
\begin{gathered}
\dot{x}_{1}(t)=16 x_{1}(t) x_{2}(t)+2 x_{1}\left(t-\tau_{1}(t)\right)-0.5 \sin \left(x_{1}\right) \cos (t), \\
\dot{x}_{2}(t)=-x_{1}(t)-x_{2}(t)+\left(x_{1}(t)+x_{2}(t)\right) x_{3}(t) \\
\quad+x_{1}\left(t-\tau_{1}(t)\right) x_{2}\left(t-\tau_{2}(t)\right)-0.5 \sin (10 t), \\
\dot{x}_{3}(t)=10\left(x_{2}(t)-x_{3}^{3}(t)+0.143 x_{3}(t)\right)+\sin (5 t) \\
+x_{1}\left(t-\tau_{1}(t)\right)+x_{3}\left(t-\tau_{3}(t)\right)+D^{3}(u(t)) \\
+\left(1+x_{2}^{2}(t)\right) D(u(t))+\sin (0.1 D(u(t))) \\
y(t)=x_{1}(t)
\end{gathered}
$$




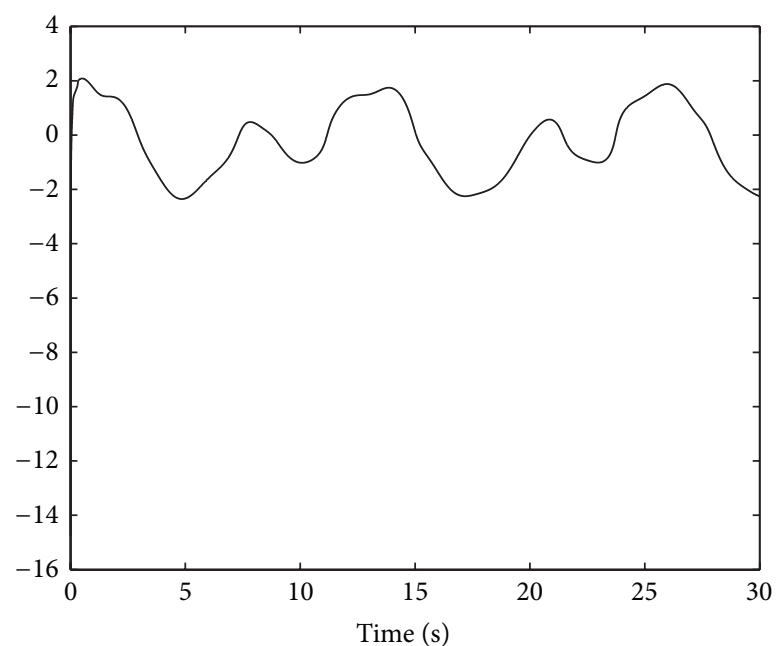

Figure 3: Control input $u$.

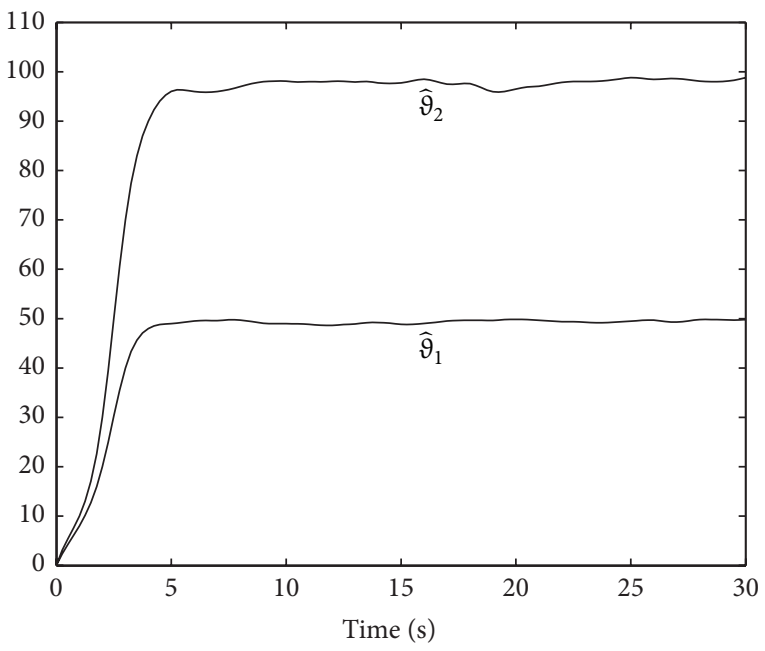

FIGURE 4: The curve of adaptive parameters $\widehat{\vartheta}_{1}$ and $\widehat{\vartheta}_{2}$.

where $\tau_{1}(t)=0.2(1+\cos (t)), \tau_{2}(t)=0.3(1+\sin (t))$, and $\tau_{3}(t)=0.1(0.5+0.2 \cos (t))$.

Similar to Example 1, the control laws and the adaptive laws are chosen as

$$
\begin{gathered}
\alpha_{1}=\frac{q_{1}\left(\chi_{1}\right)}{2 a_{1}^{2}} N\left(\varsigma_{1}\right) \chi_{1} \widehat{\vartheta}_{1}, \quad \alpha_{2}=\frac{q_{2}\left(\chi_{2}\right)}{2 a_{2}^{2}} N\left(\varsigma_{2}\right) \chi_{2} \widehat{\vartheta}_{2}, \\
u=\frac{q_{3}\left(\chi_{3}\right)}{2 a_{3}^{2}} N\left(\varsigma_{3}\right) \chi_{3} \widehat{\vartheta}_{3}, \quad \dot{\varsigma}_{1}=\frac{q_{1}\left(\chi_{1}\right)}{2 a_{1}^{2}} \chi_{1}^{2} \widehat{\vartheta}_{1}, \\
\dot{\widehat{\vartheta}}_{1}=\frac{\gamma_{1}}{2 a_{1}^{2}} \chi_{1}^{2}-\eta_{1} \widehat{\vartheta}_{1}, \quad \dot{\zeta}_{2}=\frac{q_{2}\left(\chi_{2}\right)}{2 a_{2}^{2}} \chi_{2}^{2} \widehat{\vartheta}_{2}, \\
\dot{\hat{\vartheta}}_{2}=\frac{\gamma_{2}}{2 a_{2}^{2}} \chi_{2}^{2}-\eta_{2} \widehat{\vartheta}_{2}, \quad \dot{\zeta}_{3}=\frac{q_{3}\left(\chi_{3}\right)}{2 a_{3}^{2}} \chi_{3}^{2} \widehat{\vartheta}_{3}, \\
\dot{\widehat{\vartheta}}_{3}=\frac{\gamma_{3}}{2 a_{3}^{2}} \chi_{3}^{2}-\eta_{3} \widehat{\vartheta}_{3} .
\end{gathered}
$$

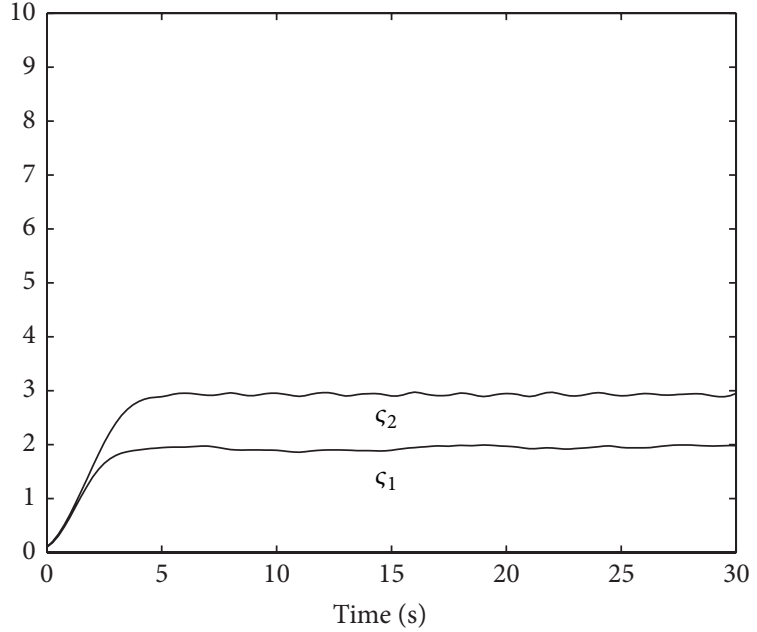

FIGURE 5: The curve of adaptive parameters $\varsigma_{1}$ and $\varsigma_{2}$.

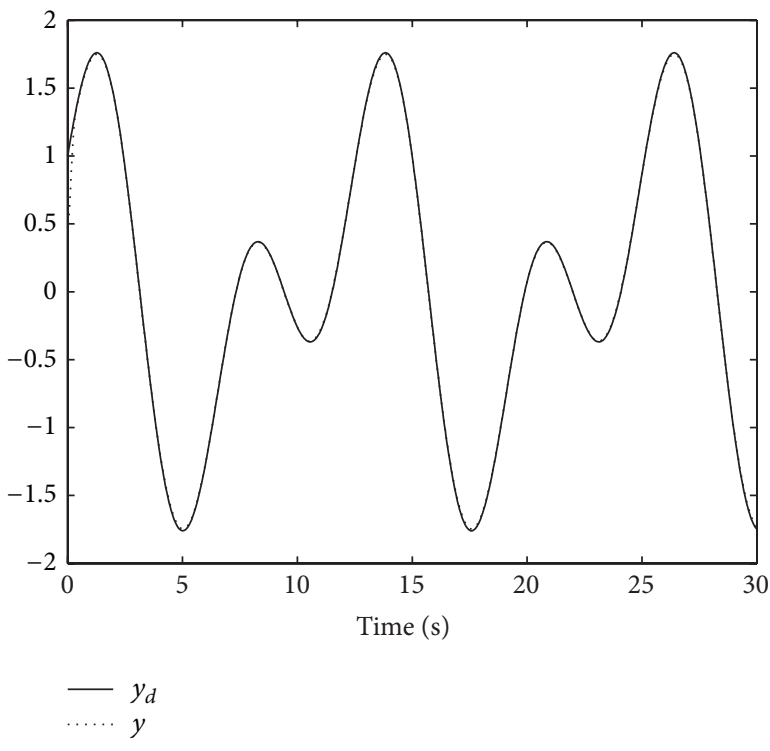

FIGURE 6: Trajectories of system output $y$ and reference signal $y_{d}$.

In this example, we choose the design parameters as $a_{1}=$ $0.2, a_{2}=0.2, a_{3}=0.2, \gamma_{1}=20, \gamma_{2}=20, \gamma_{3}=20, \eta_{1}=0.1$, $\eta_{2}=0.1, \eta_{3}=0.1, \kappa_{1}=0.1, \kappa_{2}=0.1$, and $\kappa_{3}=0.1$. The initial values are set to be $x_{1}(\omega)=0.1, x_{2}(\omega)=0$, and $x_{3}(\omega)=0$ for $\omega \leq 0, \varsigma_{1}(0)=0.01, \varsigma_{2}(0)=0.01, \varsigma_{3}(0)=0.01, \widehat{\vartheta}_{1}(0)=0.01$, $\widehat{\vartheta}_{2}(0)=0.01$, and $\widehat{\vartheta}_{3}(0)=0.01$. The simulation results are shown in Figures 6-11, respectively.

From Figure 6, it can be concluded that a good tracking performance is obtained. Figures 7 and 8 show the trajectory of state variables $x_{2}$ and $x_{3}$, respectively. Figure 9 depicts the curve of the control input signal. Figures 10 and 11 display the adaptive parameters $\widehat{\vartheta}_{1}, \widehat{\vartheta}_{2}, \widehat{\vartheta}_{3}$, and $\varsigma_{1}, \varsigma_{2}, \varsigma_{3}$, respectively.

From the simulation results, it is seen that fairly good tracking performances are achieved; meanwhile, all the other signals in closed-loop system are bounded. 


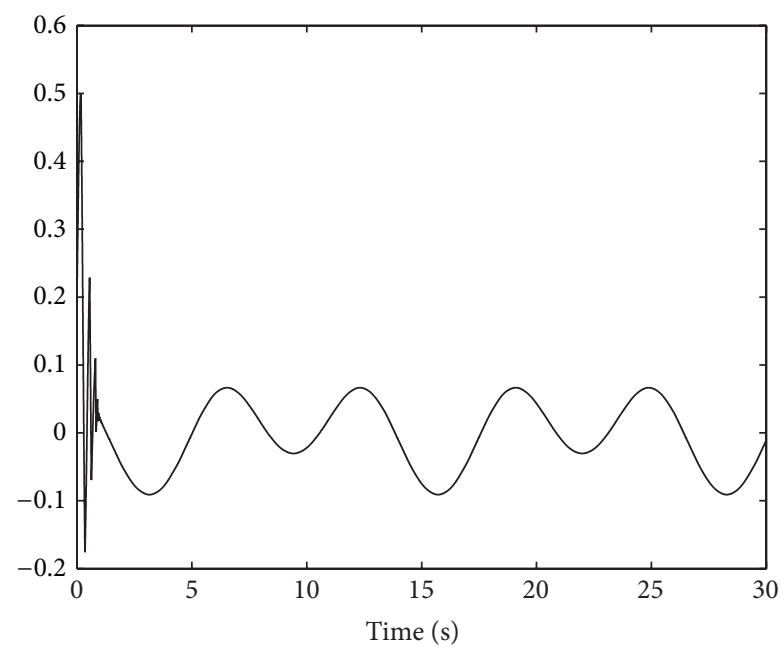

Figure 7: Trajectory of the system state $x_{2}$.

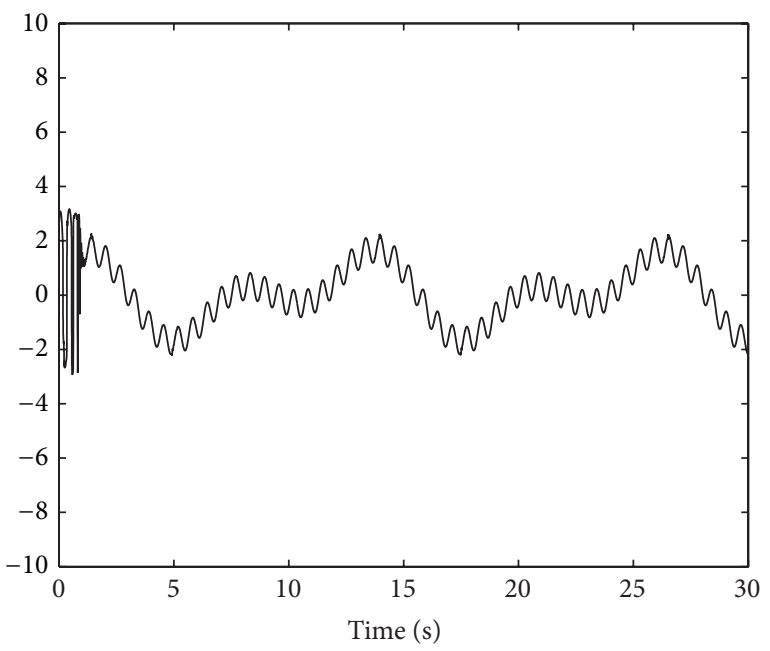

FIGURE 8: Trajectory of the system state $x_{3}$.

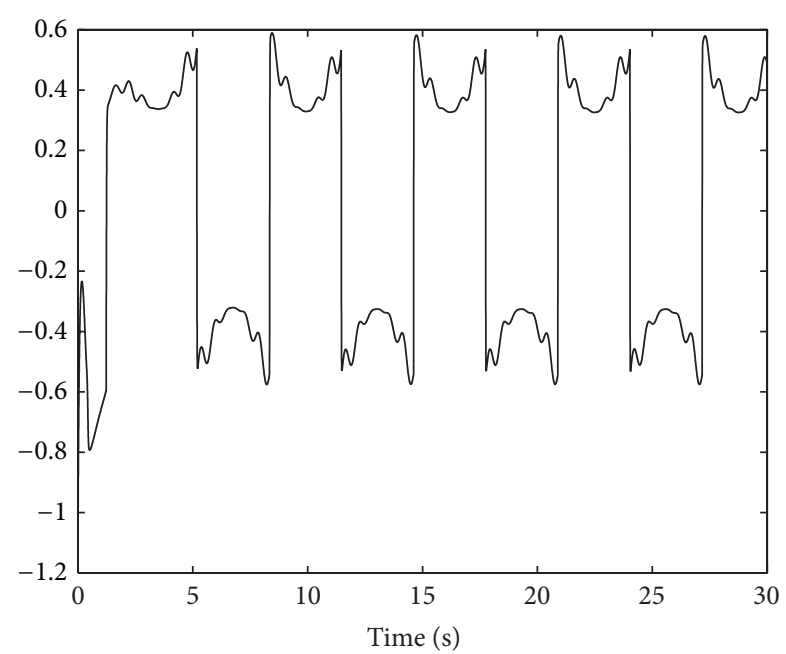

Figure 9: Control input $u$.

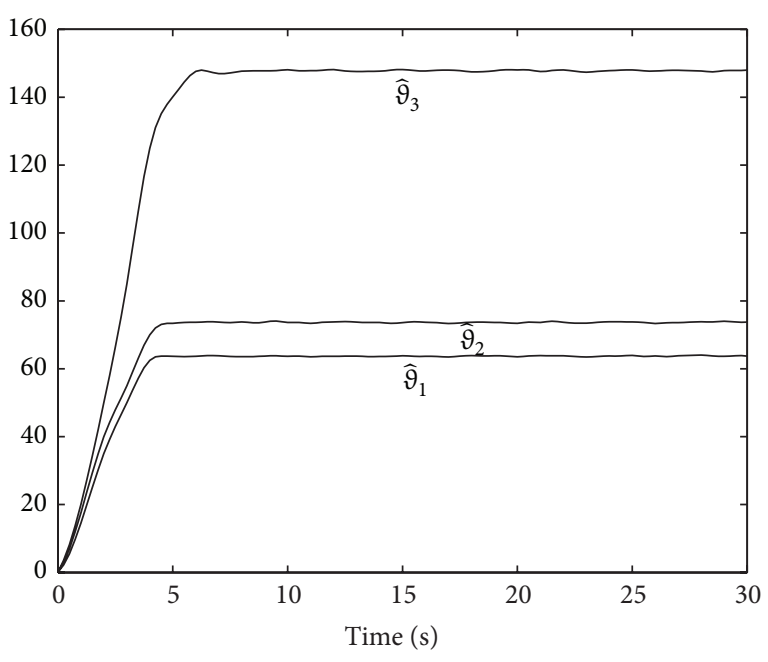

FIGURE 10: The curve of adaptive parameters $\widehat{\vartheta}_{1}, \widehat{\vartheta}_{2}$, and $\widehat{\vartheta}_{3}$.

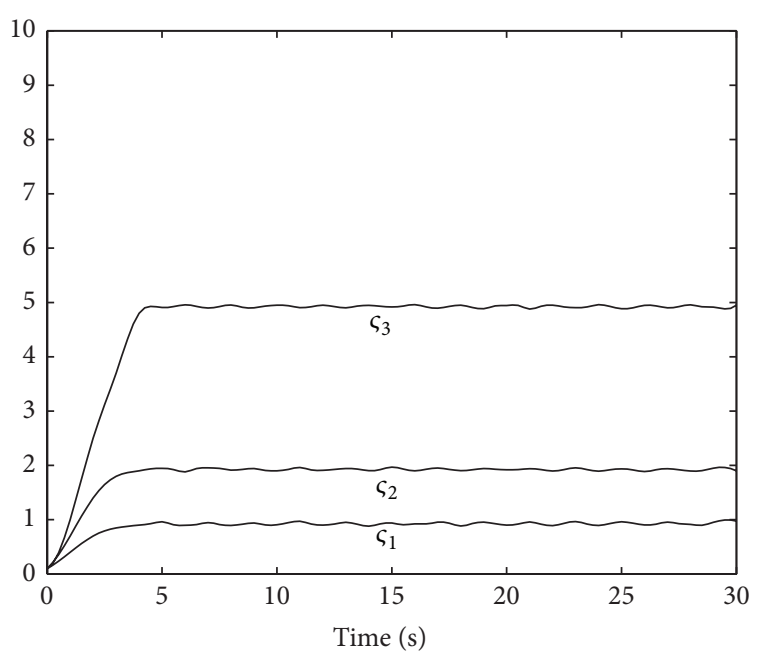

FIGURE 11: The curve of adaptive parameters $\varsigma_{1}, \varsigma_{2}$, and $\varsigma_{3}$.

\section{Conclusions}

In this paper, an adaptive fuzzy backstepping control scheme is presented for a class of nonaffine nonlinear time-delay systems with unknown control direction and unknown deadzone input nonlinearity. By choosing appropriate LyapunovKrasovskii functionals, the adaptive fuzzy controller is designed based on backstepping technique and FLSs. The proposed controller guarantees that all the signals in the closed-loop system are bounded and the tracking error eventually converges to a small neighbourhood of the origin. In addition, the number of the parameters which need to be tuned online is significantly reduced. This makes our scheme easily realized in practice. The simulation results illustrate the effectiveness and feasibility of the proposed approach. 


\section{Conflict of Interests}

The authors declare that there is no conflict of interests regarding the publication of this paper.

\section{References}

[1] I. Kanellakopoulos, P. V. Kokotović, and A. S. Morse, "Systematic design of adaptive controllers for feedback linearizable systems," IEEE Transactions on Automatic Control, vol. 36, no. 11, pp. 1241-1253, 1991.

[2] J. Zhou, C. Wen, and Y. Zhang, "Adaptive output control of nonlinear systems with uncertain dead-zone nonlinearity," IEEE Transactions on Automatic Control, vol. 51, no. 3, pp. 504511, 2006.

[3] J. Zhou and X. Z. Shen, "Robust adaptive control of nonlinear uncertain plants with unknown dead-zone," IET Control Theory \& Applications, vol. 1, no. 1, pp. 25-32, 2007.

[4] C. Hua, G. Feng, and X. Guan, "Robust controller design of a class of nonlinear time delay systems via backstepping method," Automatica, vol. 44, no. 2, pp. 567-573, 2008.

[5] S. J. Yoo, J. B. Park, and Y. H. Choi, "Adaptive dynamic surface control for stabilization of parametric strict-feedback nonlinear systems with unknown time delays," IEEE Transactions on Automatic Control, vol. 52, no. 12, pp. 2360-2364, 2007.

[6] J. A. Farrell, M. Polycarpou, M. Sharma, and W. Dong, "Command filtered backstepping," IEEE Transactions on Automatic Control, vol. 54, no. 6, pp. 1391-1395, 2009.

[7] W. Dong, J. A. Farrell, M. M. Polycarpou, V. Djapic, and M. Sharma, "Command filtered adaptive backstepping," IEEE Transactions on Control Systems Technology, vol. 20, no. 3, pp. 566-580, 2012.

[8] Y. S. Yang, G. Feng, and J. Ren, "A combined backstepping and small-gain approach to robust adaptive fuzzy control for strictfeedback nonlinear systems," IEEE Transactions on Systems, Man, and Cybernetics A:Systems and Humans, vol. 34, no. 3, pp. 406-420, 2004.

[9] Y. S. Yang and C. Zhou, "Adaptive fuzzy $\mathrm{H}_{\infty}$ stabilization for strict-feedback canonical nonlinear systems via backstepping and small-gain approach," IEEE Transactions on Fuzzy Systems, vol. 13, no. 1, pp. 104-114, 2005.

[10] B. Chen, X. Liu, K. Liu, and C. Lin, "Direct adaptive fuzzy control of nonlinear strict-feedback systems," Automatica, vol. 45, no. 6, pp. 1530-1535, 2009.

[11] W. Chen, L. Jiao, R. Li, and J. Li, "Adaptive backstepping fuzzy control for nonlinearly parameterized systems with periodic disturbances," IEEE Transactions on Fuzzy Systems, vol. 18, no. 4, pp. 674-685, 2010.

[12] H. Lee, "Robust adaptive fuzzy control by backstepping for a class of MIMO nonlinear systems," IEEE Transactions on Fuzzy Systems, vol. 19, no. 2, pp. 265-275, 2011.

[13] C. Ren, S. Tong, and Y. Li, "Fuzzy adaptive high-gain-based observer backstepping control for SISO nonlinear systems with dynamical uncertainties," Nonlinear Dynamics, vol. 67, no. 2, pp. 941-955, 2012.

[14] Q. Zhou, P. Shi, J. Lu, and S. Xu, "Adaptive output-feedback fuzzy tracking control for a class of nonlinear systems," IEEE Transactions on Fuzzy Systems, vol. 19, no. 5, pp. 972-982, 2011.

[15] S. C. Tong and Y. M. Li, "Adaptive fuzzy output feedback tracking backstepping control of strict-feedback nonlinear systems with unknown dead zones," IEEE Transactions on Fuzzy Systems, vol. 20, no. 1, pp. 168-180, 2012.
[16] S. C. Tong and Y. M. Li, "Adaptive fuzzy output feedback control of MIMO nonlinear systems with unknown dead-zone inputs," IEEE Transactions on Fuzzy Systems, vol. 21, no. 1, pp. 134-146, 2013.

[17] J. Wang and J. Hu, "Robust adaptive neural control for a class of uncertain non-linear time-delay systems with unknown deadzone non-linearity," IET Control Theory \& Applications, vol. 5, no. 15, pp. 1782-1795, 2011.

[18] X. Q. Li, D. Wang, T. S. Li, Z. Peng, G. Sun, and N. Wang, "Adaptive NN control of uncertain nonaffine pure-feedback systems with unknown time-delay," in Proceedings of the American Control Conference (ACC '11), pp. 4219-4224, July 2011.

[19] M. Wang, X. Liu, and P. Shi, "Adaptive neural control of purefeedback nonlinear time-delay systems via dynamic surface technique," IEEE Transactions on Systems, Man, and Cybernetics B: Cybernetics, vol. 41, no. 6, pp. 1681-1692, 2011.

[20] Y. Li, C. Ren, and S. Tong, "Adaptive fuzzy backstepping output feedback control for a class of MIMO time-delay nonlinear systems based on high-gain observer," Nonlinear Dynamics, vol. 67, no. 2, pp. 1175-1191, 2012.

[21] B. Chen, X. Liu, K. Liu, and C. Lin, "Adaptive fuzzy tracking control of nonlinear MIMO systems with time-varying delays," Fuzzy Sets and Systems, vol. 217, pp. 1-21, 2013.

[22] B. Chen, X. Liu, K. Liu, and C. Lin, "Adaptive control for nonlinear MIMO time-delay systems based on fuzzy approximation," Information Sciences, vol. 222, pp. 576-592, 2013.

[23] Q. Zhou, P. Shi, S. Y. Xu, and H. Y. Li, "Adaptive output feedback control for nonlinear time-delay systems by fuzzy approximation approach," IEEE Transactions on Fuzzy Systems, vol. 21, no. 2, pp. 301-312, 2013.

[24] Y. Wen and X. Ren, "Observer-based fuzzy adaptive control for non-linear time-varying delay systems with unknown control direction," IET Control Theory \& Applications, vol. 4, no. 12, pp. 2757-2769, 2010.

[25] H. Yue and J. Li, "Output-feedback adaptive fuzzy control for a class of non-linear time-varying delay systems with unknown control directions," IET Control Theory \& Applications, vol. 6, no. 9, pp. 1266-1280, 2012.

[26] S. Tong, C. Liu, and Y. Li, "Adaptive fuzzy backstepping output feedback control for strict feedback nonlinear systems with unknown sign of high-frequency gain," Neurocomputing, vol. 77, no. 1, pp. 58-70, 2012.

[27] H. Yue and J. Li, "Output-feedback adaptive fuzzy control for a class of nonlinear systems with input delay and unknown control directions," Journal of the Franklin Institute. Engineering and Applied Mathematics, vol. 350, no. 1, pp. 129-154, 2013.

[28] X.-S. Wang, C.-Y. Su, and H. Hong, "Robust adaptive control of a class of nonlinear systems with unknown dead-zone," Automatica, vol. 40, no. 3, pp. 407-413, 2004.

[29] S. Ibrir, W. F. Xie, and C.-Y. Su, "Adaptive tracking of nonlinear systems with non-symmetric dead-zone input," Automatica, vol. 43, no. 3, pp. 522-530, 2007.

[30] C.-C. Hua, Q.-G. Wang, and X.-P. Guan, "Adaptive tracking controller design of nonlinear systems with time delays and unknown dead-zone input," IEEE Transactions on Automatic Control, vol. 53, no. 7, pp. 1753-1759, 2008.

[31] H. Adloo, N. Noroozi, and P. Karimaghaee, "Observer-based model reference adaptive control for unknown time-delay chaotic systems with input nonlinearity," Nonlinear Dynamics, vol. 67, no. 2, pp. 1337-1356, 2012. 
[32] J.-Z. Kim, J.-H. Park, S.-W. Lee, and E. K. P. Chong, "Twolayered fuzzy logic controller for systems with deadzones," IEEE Transactions on Industrial Electronics, vol. 41, no. 2, pp. 155-162, 1994.

[33] F. L. Lewis, W. K. Tim, L.-Z. Wang, and Z. X. Li, “Deadzone compensation in motion control systems using adaptive fuzzy logic control,' IEEE Transactions on Control Systems Technology, vol. 7, no. 6, pp. 731-742, 1999.

[34] J. O. Jang, "A deadzone compensator of a dc motor system using fuzzy logic control," IEEE Transactions on Systems, Man and Cybernetics C: Applications and Reviews, vol. 31, no. 1, pp. 4248, 2001.

[35] H. Cho and E.-W. Bai, "Convergence results for an adaptive dead zone inverse," International Journal of Adaptive Control and Signal Processing, vol. 12, no. 5, pp. 451-466, 1998.

[36] A. Taware, G. Tao, and C. Teolis, "Design and analysis of a hybrid control scheme for sandwich nonsmooth nonlinear systems," IEEE Transactions on Automatic Control, vol. 47, no. 1, pp. 145-150, 2002.

[37] M. Ghanes, J. De Leon, and J.-P. Barbot, "Observer design for nonlinear systems under unknown time-varying delays," IEEE Transactions on Automatic Control, vol. 58, no. 6, pp. 1529-1534, 2013.

[38] X. Jiao and T. Shen, "Adaptive feedback control of nonlinear time-delay systems: the LaSalle-Razumikhin-based approach," IEEE Transactions on Automatic Control, vol. 50, no. 11, pp. 1909-1913, 2005.

[39] Z. Yu, Z. Jin, and H. Du, "Adaptive neural control for a class of non-affine stochastic non-linear systems with time-varying delay: a Razumikhin-Nussbaum method," IET Control Theory \& Applications, vol. 6, no. 1, pp. 14-23, 2012.

[40] T. M. Apostol, Mathematical Analysis, Addison-Wesley, Reading, Mass, USA, 2nd edition, 1974.

[41] L. X. Wang, A Course in Fuzzy Systems and Control, Prentice Hall, Englewood Cliffs, NJ, USA, 1997.

[42] R. D. Nussbaum, "Some remarks on a conjecture in parameter adaptive control," Systems \& Control Letters, vol. 3, no. 5, pp. 243-246, 1983. 


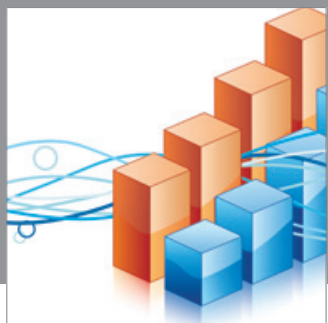

Advances in

Operations Research

mansans

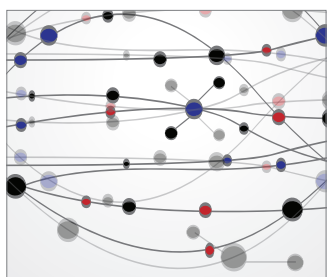

The Scientific World Journal
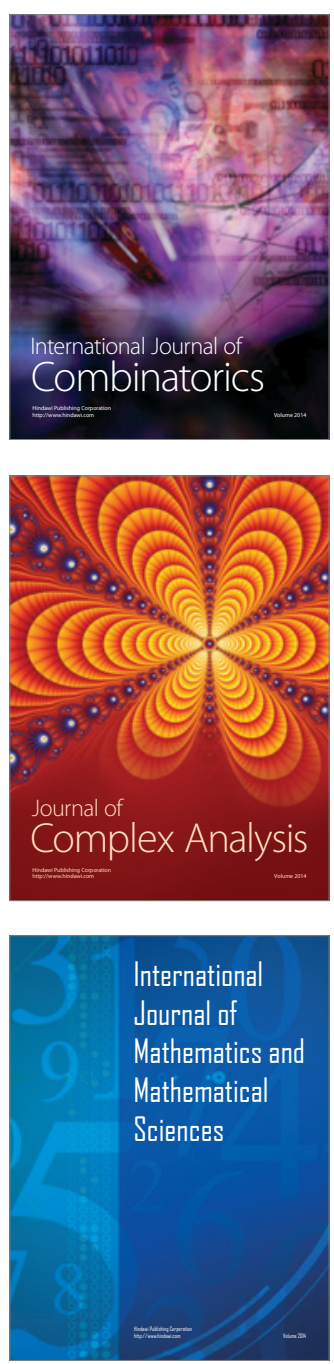
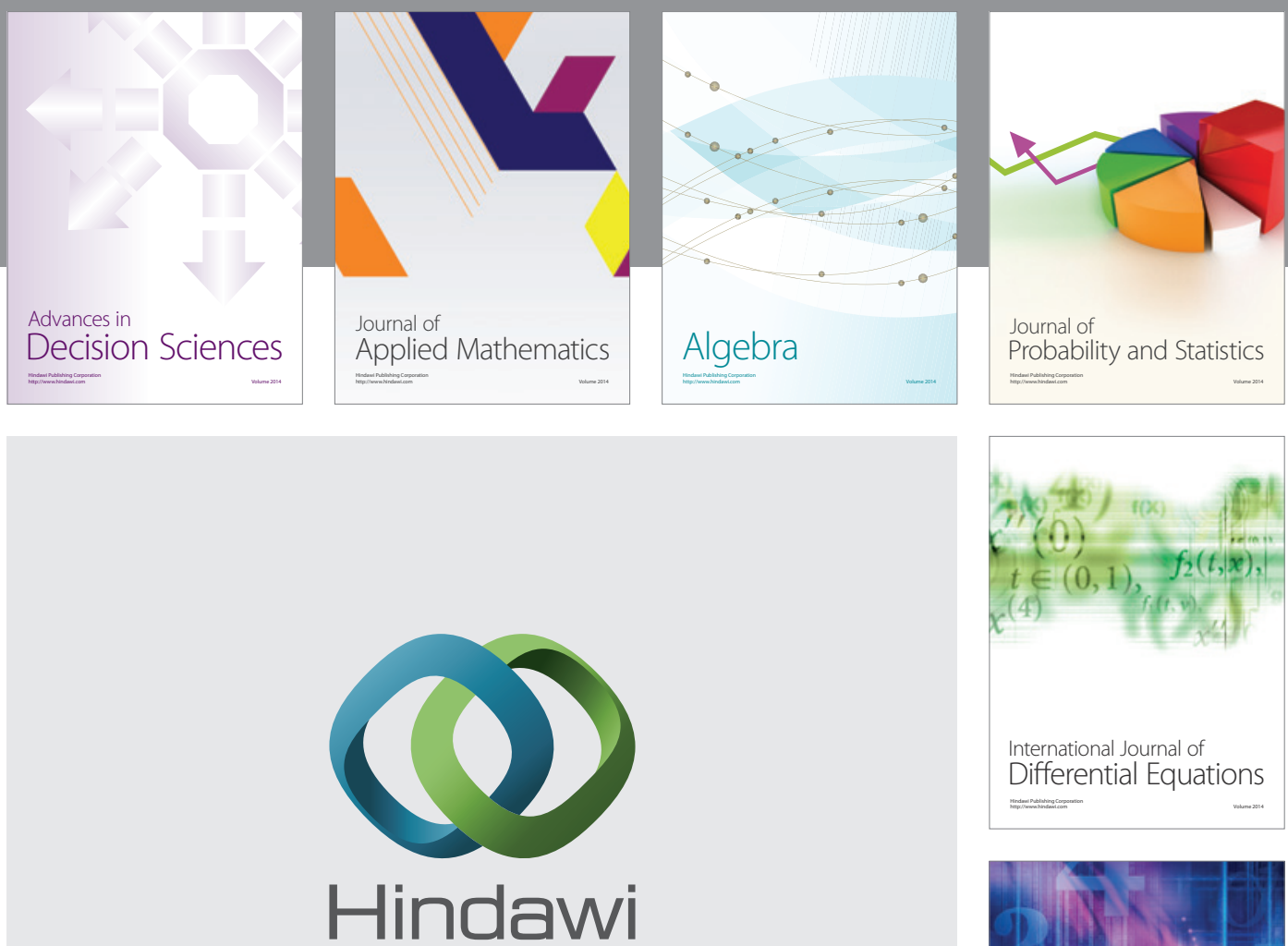

Submit your manuscripts at http://www.hindawi.com
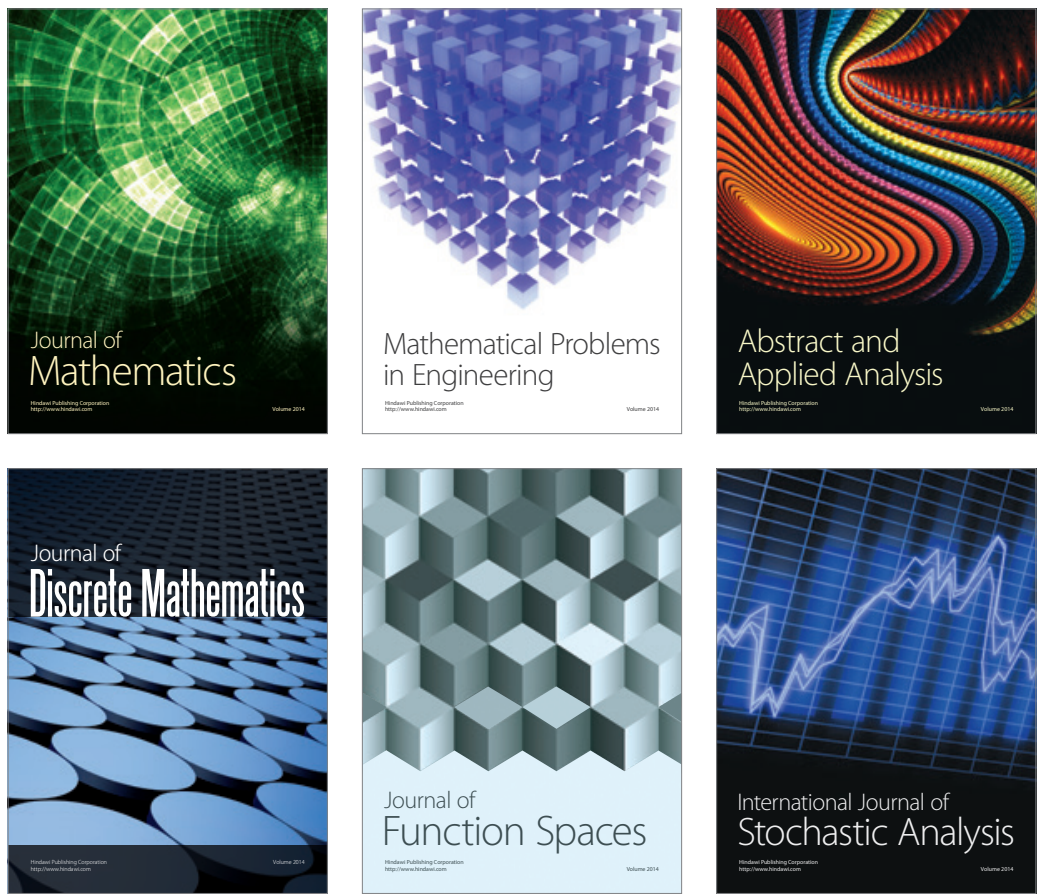

Journal of

Function Spaces

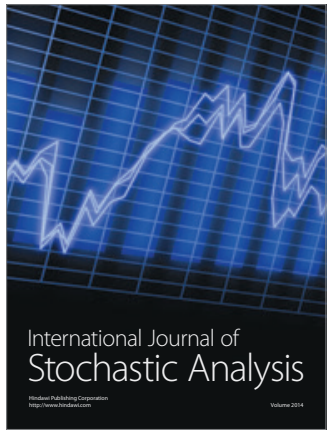

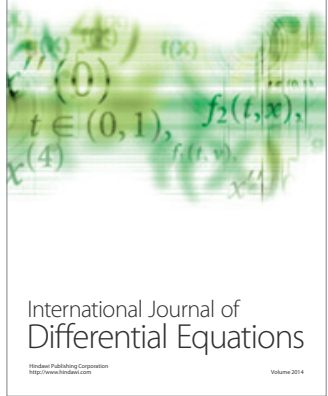
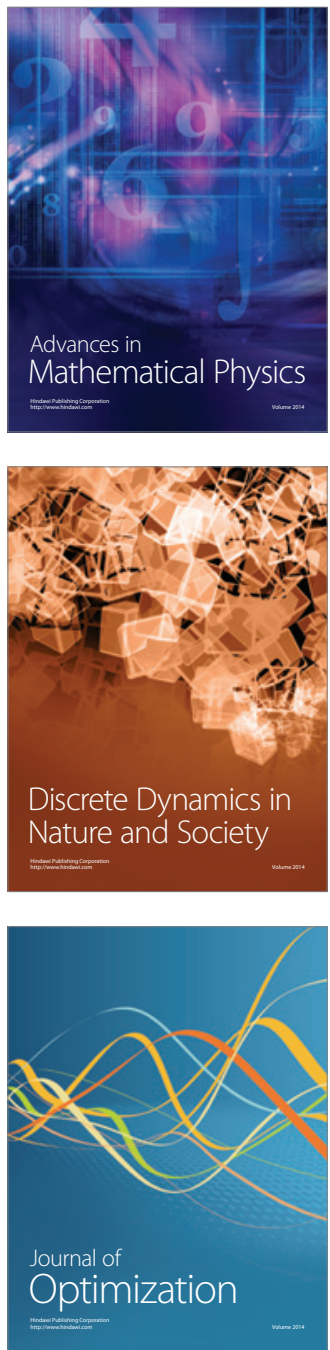